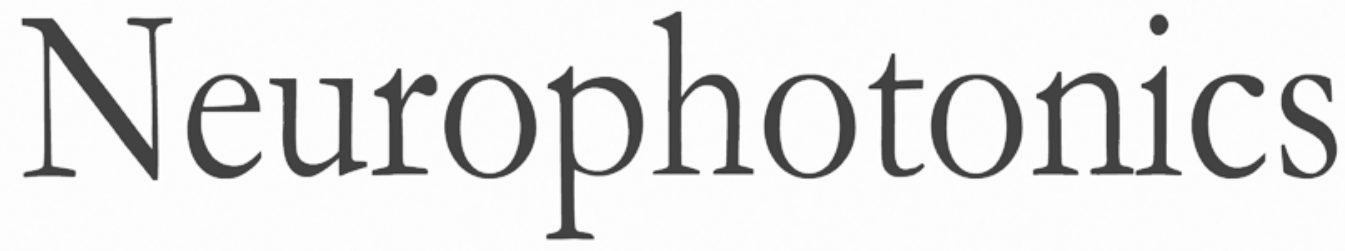

\title{
Fluorescence lifetime imaging nanoscopy for measuring Förster resonance energy transfer in cellular nanodomains
}

Christian Tardif

Gabriel Nadeau

Simon Labrecque

Daniel Côté

Flavie Lavoie-Cardinal

Paul De Koninck 


\title{
Fluorescence lifetime imaging nanoscopy for measuring Förster resonance energy transfer in cellular nanodomains
}

\author{
Christian Tardif, ${ }^{a}$ Gabriel Nadeau, ${ }^{a}$ Simon Labrecque, ${ }^{a}$ Daniel Côté,,${ }^{a, b}$ Flavie Lavoie-Cardinal, ${ }^{\text {a }}$ and \\ Paul De Koninck ${ }^{a, c, *}$ \\ ${ }^{a}$ CERVO Brain Research Center, Québec (QC), Canada \\ bUniversité Laval, Département de physique, de génie physique et d'optique, Québec (QC), Canada \\ 'Université Laval, Département de biochimie, de microbiologie et de bio-informatique, Québec (QC), Canada
}

\begin{abstract}
Microscopy methods used to measure Förster resonance energy transfer (FRET) between fluorescently labeled proteins can provide information on protein interactions in cells. However, these methods are diffraction-limited, thus do not enable the resolution of the nanodomains in which such interactions occur in cells. To overcome this limitation, we assess FRET with an imaging system combining fluorescence lifetime imaging microscopy with stimulated emission depletion, termed fluorescence lifetime imaging nanoscopy (FLIN). The resulting FRET-FLIN approach utilizes immunolabeling of proteins in fixed cultured neurons. We demonstrate the capacity to discriminate nanoclusters of synaptic proteins exhibiting variable degrees of interactions with labeled binding partners inside dendritic spines of hippocampal neurons. This method enables the investigation of FRET within nanodomains of cells, approaching the scale of molecular signaling. $\odot$ The Authors. Published by SPIE under a Creative Commons Attribution 4.0 Unported License. Distribution or reproduction of this work in whole or in part requires full attribution of the original publication, including its DOI. [DOI: 10.1117/1.NPh.6.1.015002]
\end{abstract}

Keywords: stimulated emission depletion; synaptic signaling; fluorescence lifetime imaging microscopy; synaptic proteins; glutamate receptors; CaMKII.

Paper 18045R received Jul. 27, 2018; accepted for publication Nov. 28, 2018; published online Jan. 31, 2019.

\section{Introduction}

To understand cell signaling at the molecular level, the capacity to monitor and resolve molecular interactions at their scale is mandatory. Förster resonance energy transfer (FRET), a physical nonradiative process that occurs between an excited fluorophore (donor) and another fluorophore (acceptor), can be measured to evaluate molecular proximity. Combined to molecular spectroscopy or optical imaging techniques, it allows accurate distance measurements between interacting molecules at the nanoscale $(<10 \mathrm{~nm})$. Fluorescence lifetime imaging microscopy (FLIM) provides a sensitive approach to measure FRET, by quantifying the decrease in the lifetime of the donor fluorophore when an acceptor fluorophore is within $\sim 10 \mathrm{~nm} .{ }^{1,2}$ FRET-FLIM allow spatial distribution measurements of molecule ensembles within several structural states; however, the conventional microscopy methods used for FLIM are diffraction-limited, limiting the resolution of these measurements. Single-molecule FRET is a well-established method to study single protein conformation and dynamics. ${ }^{3-5}$ FRETFLIM measurements combined with single-molecule microscopy were successfully implemented. ${ }^{6}$ FRET-FLIM was also combined with structured illumination microscopy. ${ }^{7}$

Optical nanoscopy methods that overcome the diffraction barrier such as stimulated emission depletion (STED) ${ }^{8}$ allow precise characterization of the spatial distribution and organization of cellular nanodomains. FLIM has been successfully combined with STED nanoscopy in fixed biological samples ${ }^{9-11}$

*Address all correspondence to Paul De Koninck, E-mail: paul.dekoninck@ neurosciences.ulaval.ca and reversible saturable optical linear fluorescence transitions (RESOLFT) for live-cell imaging. ${ }^{12}$

In this study, we evaluated the benefits of performing FRET with fluorescence lifetime imaging nanoscopy (FRETFLIN) over FRET-FLIM, using simulation, and compared different fluorescence lifetime analysis approaches. We performed FRET-FLIN on a custom-built STED microscope to spatially resolve protein interactions at the nanoscale in dendritic spines of cultured hippocampal neurons. Dendritic spines are 0.5 to $2 \mu \mathrm{m}$ in size; they decorate dendrites and constitute the main sites of excitatory synapses on neurons. The proteins that participate in synaptic transmission in dendritic spines are densely packed, forming the postsynaptic density (PSD). To label putatively interacting proteins with donor and acceptor dyes, inside spines, we used an immunocytochemistry-based approach. Our results demonstrate the capacity of FRET-FLIN to observe signaling in nanoclusters of proteins. It can discriminate the extent of interaction that distinct synaptic receptor nanoclusters have with labeled protein partners, inside dendritic spines of hippocampal neurons.

\section{Results}

\subsection{FLIN Microscope System}

We built a STED microscope with additional time-correlated single-photon counting (TCSPC) capacity to perform FRETFLIN measurements in cultured hippocampal neurons (Sec. 4). ${ }^{10,13,14}$ This system could perform STED imaging of ATTO 594 and confocal imaging of ATTO 647N. In STED nanoscopy, the resolution is improved using a donut-shaped depletion beam that typically lasts a few hundreds of picoseconds to ensure optimal depletion of the fluorescence 
surrounding the center of the point spread function. However, for FLIN, considering the fluorescence lifetime of organic dyes or fluorescent proteins ( 1 to $5 \mathrm{~ns}$ ), keeping the pulse length short, increases the reliability of lifetime quantification. We, therefore, opted for a 150-ps excitation and depletion pulses. To characterize the resolution of our STED microscope, we measured the full width at half maximum (FWHM) on single clusters of the neuronal glutamate receptor GluN2B and antibody clusters on a glass coverslip. We could resolve nanoclusters well below the diffraction limit with a mean FWHM of $73.3 \mathrm{~nm}$ (STD $13.7 \mathrm{~nm}$ ) for GluN2B and $66.9 \mathrm{~nm}$ (STD $11.0 \mathrm{~nm}$ ) for antibody clusters (Sec. 4).

A critical requirement for fluorescence lifetime measurements is to collect sufficient photons per pixel to generate precise fitting statistics on the lifetime of the fluorophores. ${ }^{15}$ Inherent to a smaller PSF, FLIN will collect much less photons compared with FLIM. A strategy to obtain sufficient photons is to apply spatial binning. With our experimental settings (binning of 2), we collected around 14,000 photons in FLIM mode and 1000 in FLIN mode (Sec. 5). We combined the FLIN signal (pixel size of $48 \times 48 \mathrm{~nm}$ ) with the STED intensity images (pixel size of $24 \times 24 \mathrm{~nm}$ ) generating intensity-weighted lifetime images. Considering the recording times of several minutes necessary to obtain a sufficient number of photons, we performed FRET-FLIN on fixed neurons immunolabeled against the proteins of interest. ${ }^{16,17}$ This prevented live cell protein mobility and interaction variations during single-photon counting to blur the recorded FRET and intensity signals.
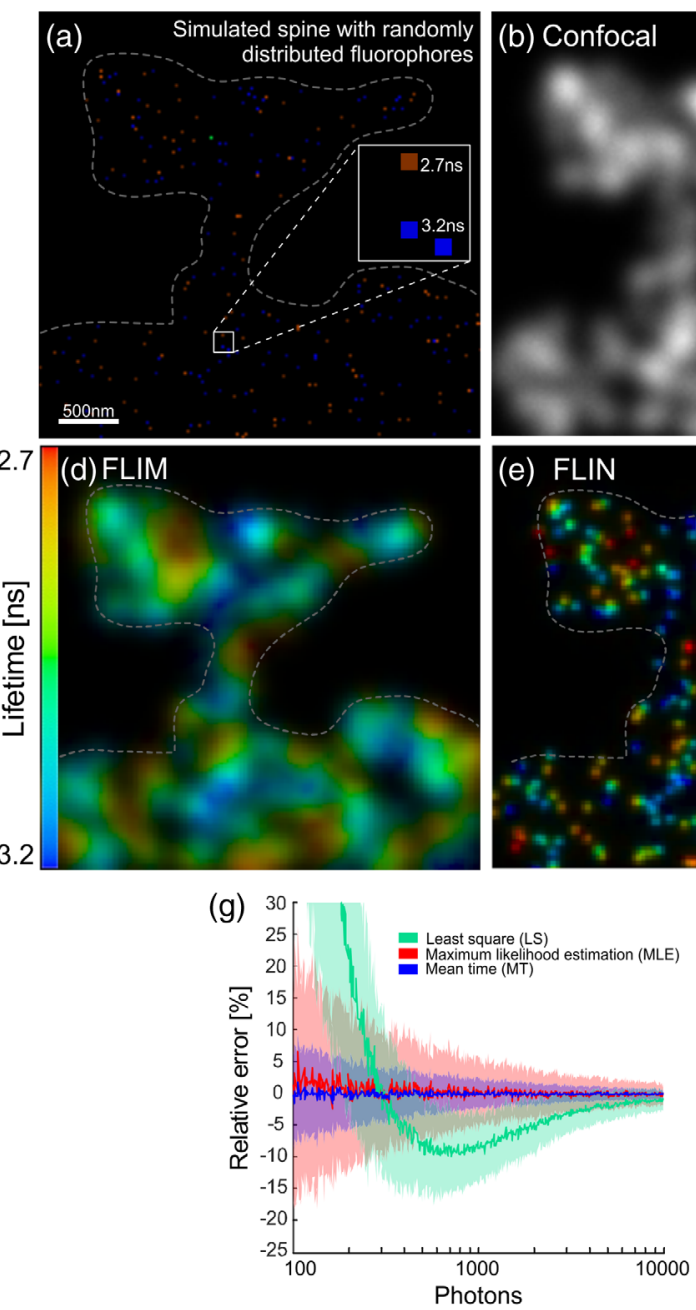
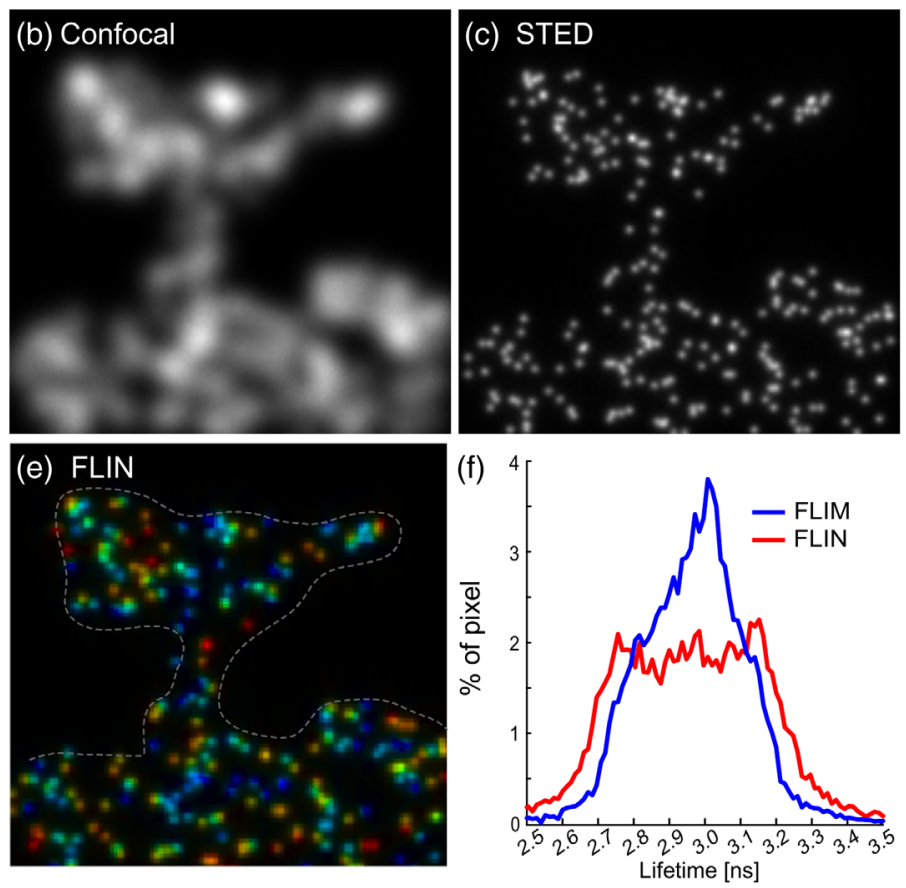

(h)

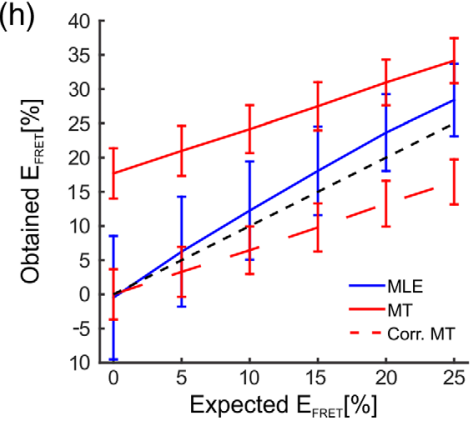

Fig. 1 Simulations of fluorescence lifetime imaging and analysis with diffraction-limited resolution $(250 \mathrm{~nm}$; FLIM) or subdiffraction-limited resolution $(50 \mathrm{~nm}$; FLIN). (a) Simulated image of randomly positioned fluorescent molecules inside a dendritic spine shape $(2.8 \times 2.0 \mu \mathrm{m})$ with a lifetime of $3.2 \mathrm{~ns}$ (donor alone) and $2.7 \mathrm{~ns}$ (corresponding to 15\% FRET efficiency). (b and c) Simulated confocal (b) and STED (c) intensity image of the molecule distribution showed in (a) (FWHM of the simulated PSFs: $250 \mathrm{~nm}$ for confocal and $50 \mathrm{~nm}$ for STED). (d and e) Simulated FLIM (d) and FLIN (e) intensity-weighted lifetime image obtained by the multiplication of the color-coded lifetime image and the simulated intensity images showed in (b) and (c), respectively. (f) Lifetime distributions for the images showed in ( $d$ and e). (g) Relative mean error ( \pm standard deviation, light color) for the fitting algorithms least square (LS), maximum likelihood (MLE), and mean time of photon arrival (MT). (h) Relationship between simulated and ground truth values of FRET efficiency, in the presence of imposed levels of $E_{\text {FRET }}$, using the MT (red), corrected MT (dash red) or MLE (blue) analysis (standard deviation indicated by the error bars). Simulated $E_{\text {FRET }}$ is indicated by the dashed black line (correlation of 1.0). 


\subsection{Simulations: FRET-FLIM versus FRET-FLIN}

We used simulations to evaluate the benefits of FRET-FLIN over FRET-FLIM, as well as the optimal parameters to use for analysis. The simulation of the fluorescence lifetime dynamics was based on the fluorescence rate equations (see Sec. 5.1). We simulated results with a (i) FLIM configuration on a diffraction limited confocal microscope (PSF $250 \mathrm{~nm}$ ) and a (ii) FLIN configuration on a super-resolution STED microscope (PSF $50 \mathrm{~nm}$ ). We randomly positioned donor fluorescent molecules without FRET interaction (lifetime of $3.2 \mathrm{~ns}$ ) or with a FRET efficiency of $15 \%$ (lifetime of $2.7 \mathrm{~ns}$ ) (see Fig. 1 and Sec. 5). FLIM would generate a blurry heat map image of lifetimes in the spine [Fig. 1(d)], as we have observed experimentally. ${ }^{18}$ FLIN would allow to resolve various lifetimes across different nanoclusters [Fig. 1(e)]. As Fig. 1(f) indicates, a wider range of lifetimes can be discriminated with FLIN compared to FLIM, due to the averaging that the lower resolution imposes in FLIM.

We explored with simulations the various methods to analyze fluorescence lifetime with limited photons available, inherent to a smaller PSF, and in the presence of FRET (see Sec. 5). To analyze the lifetime measurements, the accuracy of a commonly used method in commercial systems, the least square fit (LS), ${ }^{19,20}$ dropped dramatically with $<3000$ photons, introducing systematic errors [Fig. $1(\mathrm{~g}){ }^{21}{ }^{21}$ With low photon counts, the mean photon arrival time (MT) approach ${ }^{19,22,23}$ provides a low relative error [Fig. 1(g)], but when applied to FRET-FLIM analysis, an increasing systematic error is introduced with increasing FRET efficiency [Fig. 1(h)]. This effect can be related to the single-component lifetime assumption made in MT analysis, while FRET introduces a multicomponent lifetime. ${ }^{2,24}$ Thus, analysis using MT would underestimate the level of FRET, unless a linear correction is applied based on our simulations. The maximum likelihood fit (MLE), ${ }^{20,21}$ which is a wellestablished method for STED-FLIM analysis, ${ }^{10,12,25,26}$ do not introduce systematic errors related to FRET efficiency ${ }^{21}$ with a similar relative error but generated a slightly higher standard deviation with $<3000$ photons [Fig. $1(\mathrm{~g})$ ]. Based on these simulations, which emphasize the impact of fluorescence lifetime analysis method with limited photon collection, we chose the MLE method for FLIN data analysis, as it provides reasonable accuracy under low photon count, without the need to correct for a systematic error.

\subsection{FRET-FLIN to Resolve FRET in Nanoclusters}

To test whether we could measure FRET with FLIN in nanoclusters of immunolabeled proteins, we chose a protein complex, $\alpha \mathrm{CaMKII}$, made of 12 similar subunits (forming a holoenzyme of two opposed hexamers). Each subunit should be within approximately $5 \mathrm{~nm}$ to each other inside a CaMKII hexamer, or $\sim 8 \mathrm{~nm}$ between opposing subunits on separate hexamers. ${ }^{27}$ (a)
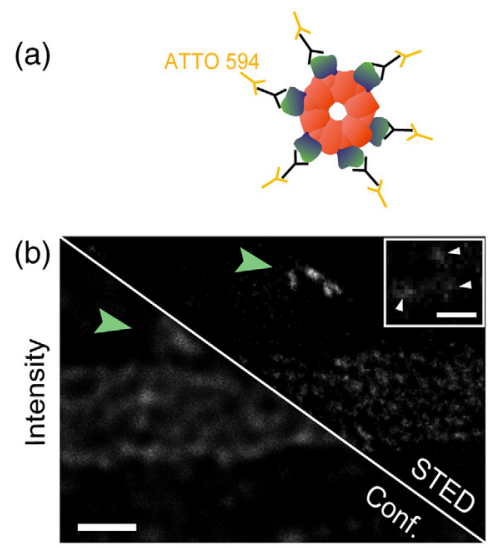

(c)
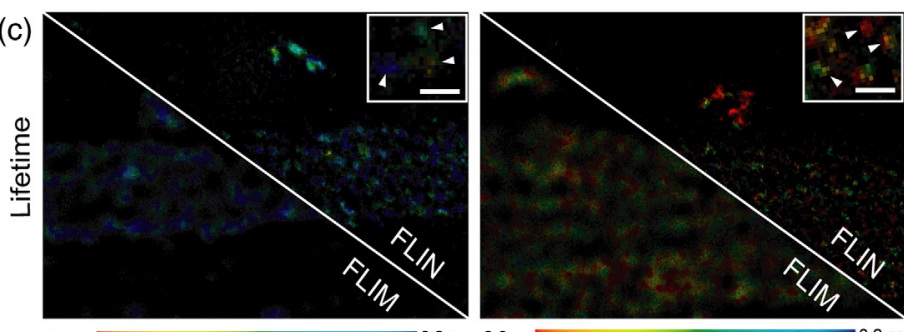

2.3

Fig. 2 FRET-FLIN on immunolabeled CaMKII nanoclusters. (a) Cartoons of $\alpha$ CaMKII labeled with mouse anti- $\alpha \mathrm{CaMKII}$ and (left) GAM-ATTO 594 (donor alone) or (right) GAM-ATTO 594 and GAM-ATTO 647N (donor + acceptor). (b) Confocal and STED intensity images of $\alpha \mathrm{CaMKII}$ in dendritic segments of cultured hippocampal neuron with (left) donor alone or (right) donor + acceptor. (c) FLIM and FLIN intensity weighted lifetime of $\alpha \mathrm{CaMKII}$ labeled with (left) the donor alone or (right) donor + acceptor. White arrows in the zoomed insets indicate the position of nanoclusters. Green arrows show dendritic spines. Scale bars: insets $250 \mathrm{~nm}$, main images $1 \mu \mathrm{m}$. Colorbar: $2.3 \mathrm{~ns}$ (red, high FRET efficiency) to $3.2 \mathrm{~ns}$ (blue, low FRET efficiency). (d) Fluorescence lifetime in nanoclusters of $\alpha$ CaMKII labeled with donor alone (3108 ps, IQR $=418 \mathrm{ps}, n=1872$ clusters, six neurons) or donor + acceptor showing a clear reduction of the measured lifetime in the presence of acceptor due to FRET ( $2442 \mathrm{ps}$, IQR $=330 \mathrm{ps}, n=1531$ clusters, seven neurons) $(p<0.001)$. The box plots here and thereon indicate first and third quartile (box edges), the median (red line), and the $\mathrm{min} / \mathrm{max}$ values (whiskers). 
$\alpha$ CaMKII was immunolabeled with a well-characterized monoclonal antibody that binds to the regulatory domain of the kinase ${ }^{28}$ which should be positioned $\sim 6.6 \mathrm{~nm}$ apart on neighboring subunits. ${ }^{27}$ Equal concentrations of donor (ATTO 594) and acceptor (ATTO 647N) secondary antibody were added [Fig 2(a)]. We expected this labeling configuration to yield strong FRET given the proximity of the donor and acceptor labeled secondary antibodies. With STED, we observed nanoclusters of immunolabeled $\alpha \mathrm{CaMKII}$ in spines and dendrites [Fig. 2(b)]. In the presence of the acceptor, FLIN measurements yielded a fluorescence lifetime around $2.4 \mathrm{~ns}$ in single nanoclusters, which is significantly shorter compared with the control experiment with donor alone $(3.1 \mathrm{~ns})$. This corresponds to an averaged FRET efficiency of $21.4 \%$. These results indicate that immunostaining can be applied with FLIN to measure FRET inside protein nanoclusters. Analysis of these FLIN measurements with MT, by comparison with MLE, yielded a smaller difference, similar to that predicted by our simulations (Sec. 5.3 and Fig. 3).

We next tested whether our approach can discriminate the proximity of two labels that are within close or distal FRET range in a dendritic spine. For these experiments, we immunolabeled two NMDA receptor subunits (GluN1 and GluN2B), which are part of the same tetrameric receptor $\left(2: 2\right.$ ratio) ${ }^{29} \mathrm{We}$ used recombinant tagged subunits (GluN1-GFP and GluN2BHA) to ensure specificity of the antibody recognition and to control epitope location [Figs. 4(a) and 4(b)]. Despite its larger size, GFP has been used as a tag on glutamate receptor subunits before, without interference on receptor expression and assembly. ${ }^{18,30,31}$ We first coexpressed GluN1-GFP and GluN2B$\mathrm{HA}$, both tags being on the c-terminus of the receptor subunits at the intracellular side of the plasma membrane, to evaluate the performance of our method when significant FRET is expected [Fig. 4(b)]. We also coexpressed untagged GluN1 and GFPGluN2B-HA constructs, with the GFP tag being extracellular and the HA-tag intracellular, to assess the performance of our

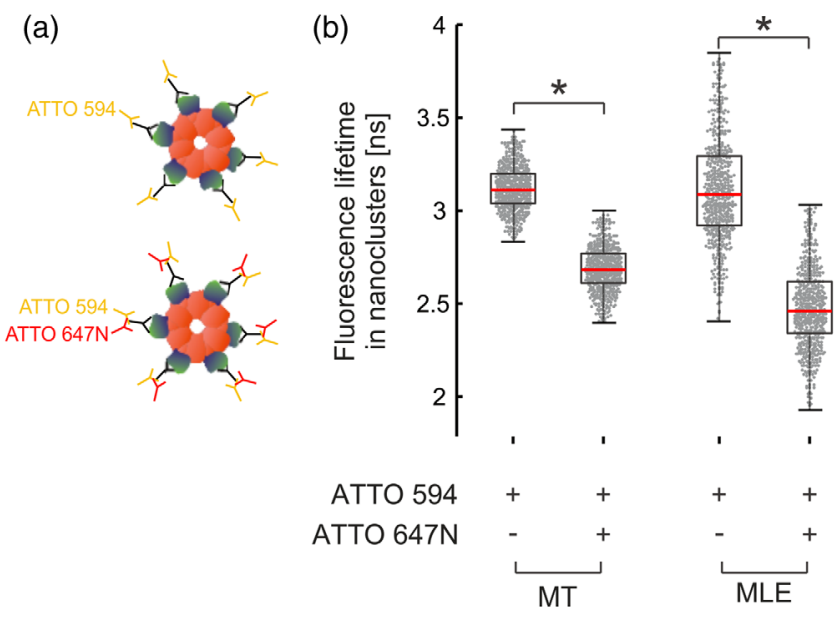

Fig. 3 Comparison between the MT and MLE analysis for the experiment shown in Fig. 2. (a) Cartoons of $\alpha$ CaMKII labeled with mouse anti- $\alpha$ CaMKII and (i) GAM-ATTO 594 (donor alone, left) or (ii) GAM-ATTO 594 and GAM-ATTO 647N (donor + acceptor, right). (b) Lifetime distributions of the dataset from Fig. 2 analyzed using the MT of photon arrival or MLE. Note the increased variability of the fluorescence lifetime values when analyzed with MLE compared with MT, while the medians are slightly more separated when analyzed with MLE, as predicted by our simulation (Fig. 1). approach when low FRET efficiency is expected [Fig. 4(a)]. We fixed the neurons with methanol and immunostained with the same pairs of primary (anti-HA and anti-GFP) and secondary (ATTO 594 and ATTO 647N) antibodies. We measured a median FRET efficiency of $3.2 \%(\mathrm{IQR}=11.1 \%$ ) in nanoclusters inside spines when both tags were on opposite sides of the receptor (GFP-GluN2B-HA). The small level of measured $E_{\text {FRET }}$ may reflect the fact that the epitopes on both ends of the GluN2B are $<10 \mathrm{~nm}$ apart. However, the distance uncertainty caused by primary/secondary antibody labeling may lead to some FRET, even if the epitopes are slightly more than $10 \mathrm{~nm}$ away. Nevertheless, the fluorescence lifetime of the donor + acceptor was not significantly different compared with donor alone (Sec. 5.3 and Fig. 12). It should be noted that the plasma membrane should have little impact on FRET, as it is largely dissolved by the methanol fixation. In contrast, for the GluN1-GFP/GluN2B-HA pair, where both tags are on the same side of the tetrameric receptor, the median FRET efficiency was significantly larger $(9.5 \%$, IQR $=9.8 \%)$ [Figs. 4 (b) and 4(c)]. These results indicate that our immuno-FRETFLIN approach can discriminate proximity of labels that are within short distances inside the FRET range.

\subsection{Monitoring CaMKII Signaling at the Nanoscale}

To assess the reliability of this approach to characterize different levels of signaling activity, we measured the changes in $\alpha$ CaMKII T286 phosphorylation using a double immunostaining of $\alpha$ CaMKII (ATTO 594-Donor) and phosphoT286$\alpha$ CaMKII (ATTO 647N-Acceptor) [Fig. 5(a)]. Neurons were fixed with or without prior bath application of a solution lacking $\mathrm{Mg}^{2+}$, containing glycine and picrotoxin (used to induce chemical long-term potentiation or cLTP). ${ }^{32}$ The rationale for this experiment was to examine whether $\alpha \mathrm{CaMKII}$ autophosphorylation, expected to occur upon cLTP stimulation, ${ }^{32}$ could be detected and localized in $\alpha$ CaMKII nanoclusters via FRETFLIN. If so, we expect higher levels of FRET on $\alpha$ CaMKII nanoclusters that include phosphorylated $\alpha$ CaMKII subunits. To set a baseline level of FRET, we incubated the neurons with NMDA receptor blocker AP5, to reduce $\alpha$ CaMKII phosphorylation at $\mathrm{T} 286 .{ }^{32}$ Under these conditions, the median FRET efficiency in $\alpha$ CaMKII nanoclusters was $3.5 \%$ (IQR 12.1) [Fig. 5(b)]. Under these conditions, the fluorescence lifetime was significantly lower to that of the donor alone (Sec. 5.3 and Fig. 12), consistent with some binding of antiphosphoT286 antibody under basal condition. After cLTP stimulation, the median FRET efficiency significantly increased to $5.2 \%$ (IQR 8.4), consistent with additional autophosphorylated $\alpha$ CaMKII. After 10-min wash in AP5 poststimulation [Fig. 5(a), 10-min post-cLTP], the median FRET efficiency dropped to $4.1 \%$ (IQR 11.3). These results indicate that immuno-FRET-FLIN can reveal changes of phosphorylation level in nanoclusters of proteins inside a single dendritic spine.

\subsection{Monitoring Interactions of $\alpha$ CaMKII with GluN2B Nanoclusters in Dendritic Spines}

We further tested if our method could be applied to measure the levels of interaction between two different proteins. Several reports have shown that $\alpha \mathrm{CaMKII}$ can interact with the c-tail of the NMDA receptor subunit GluN2B, and that this interaction is promoted by neuronal activity. ${ }^{3-37}$ For this purpose, we immunostained GluN2B with a rabbit antibody targeting its 
(a)
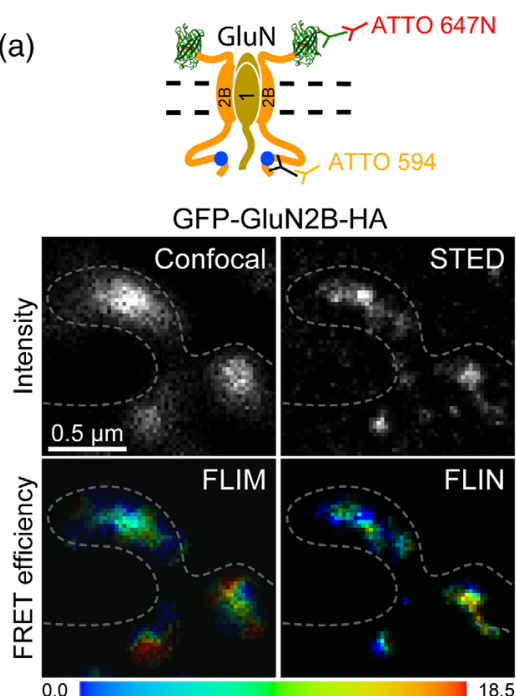

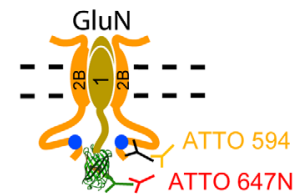

(c)

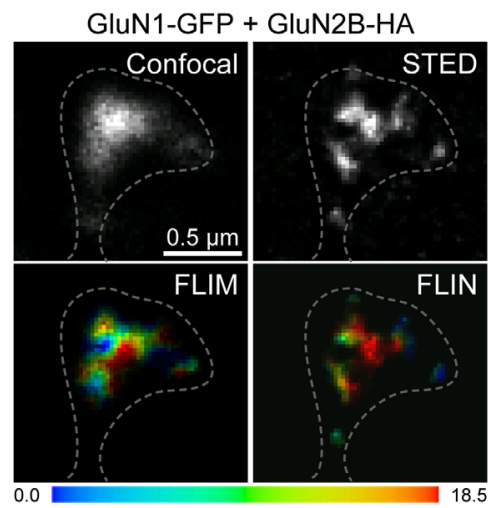

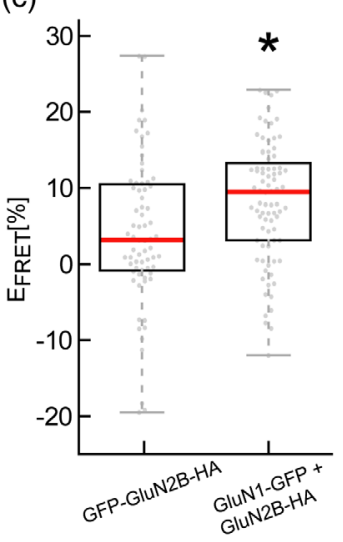

Fig. 4 Assessment of FRET-FLIN sensitivity by dual immunolabeling of NMDA receptor subunits with proximal and distal epitopes. (a, b) Two immunolabeling configurations of NMDA receptors with both the donor (anti-HA/ATTO 594) and acceptor (anti-GFP/ATTO 647N) on (a) opposite side of the plasma membrane (GFP-GluN2B-HA) or (b) on the same side of the plasma membrane (GluN1-GFP and GluN2B$H A$ ). Confocal and STED images of dendritic spines (top gray scale images) and corresponding intensityweighted lifetime images of FRET efficiencies shown with FLIM and FLIN (bottom images, color map from $0 \%$ to $18.5 \%$ FRET efficiency). (c) The median FRET efficiency per spine cluster for the configuration in (b) $(9.5 \%$, IQR $=9.8 \%, n=81$ clusters, five neurons) is significantly higher compared to that in (a) $(3.2 \%, I Q R=11.1 \%, n=69$ clusters, five neurons $)(p=0.003)$, indicating that FLIN can discriminate FRET levels between the donor and acceptor located either on the intracellular c-tails of the receptor or on opposite ends of the receptor.

(a)
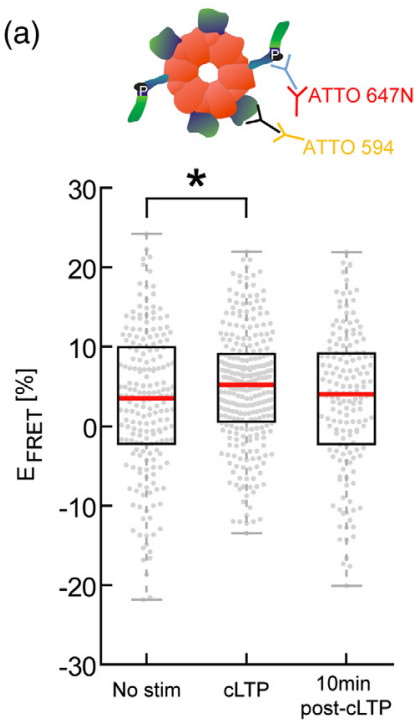

(b)
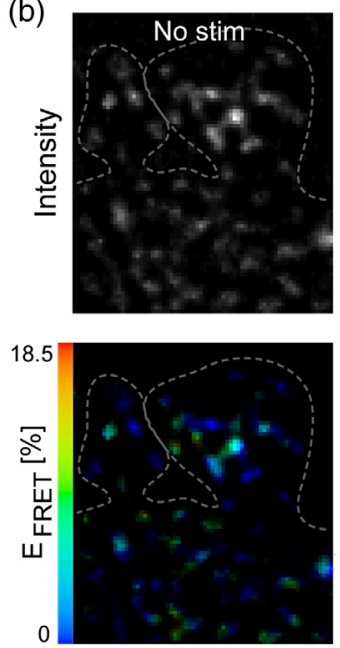
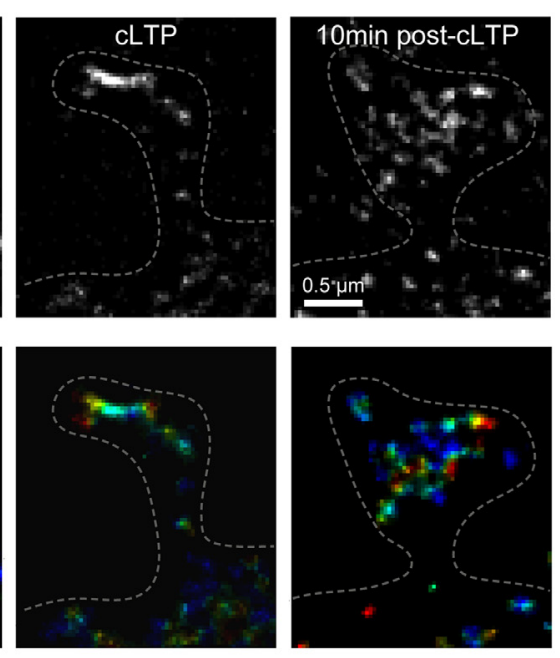

Fig. 5 Assessment of changes in CaMKII autophosphorylation by FRET-FLIN. (a) Double immunolabeling of anti- $\alpha$ CaMKII/ATTO 594 and anti-pT286-CaMKII/ATTO 647N (top cartoon). Bottom: Distribution of FRET efficiencies per nanocluster, representing T286 phosphorylation in $\alpha \mathrm{CaMKII}$, as shown in (b), for no stimulation ( 5 min preincubation in AP5) (3.5\%, IQR $=12.1 \%, n=199$ clusters, three neurons), CLTP (5 min in $0 \mathrm{Mg}^{2+}$ /glycine/picrotoxin) $(5.2 \%, \mathrm{IQR}=8.4 \%, n=280$ clusters, four neurons), and post-cLTP (10 $\mathrm{min}$ in AP5) $(4.1 \%, \mathrm{IQR}=11.3 \%, n=160$ clusters, four neurons) conditions. The data reveal heterogeneity in CaMKII autophosphorylation inside spines, with a significant increase of eFRET upon CLTP stimulation, compared with no stimulation condition $(p=0.03)$ and a partial reversibility upon washout $(p=0.63)$. (b) Top row: representative images of dendritic spines for the indicated conditions, taken with STED. Botton row: corresponding intensity weighted FLIN images showing FRET efficiencies in nanoclusters of CaMKII. 
(a)

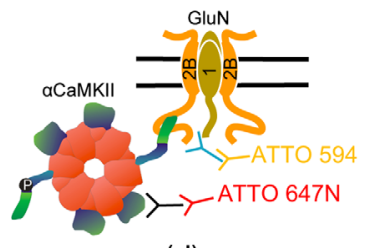

(c)

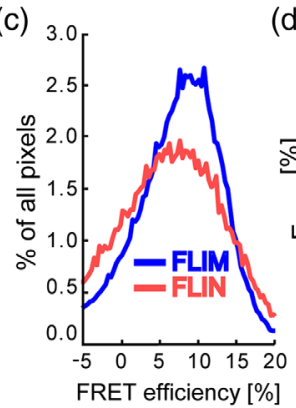

(d)

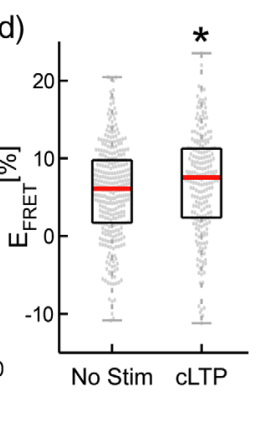

(b)

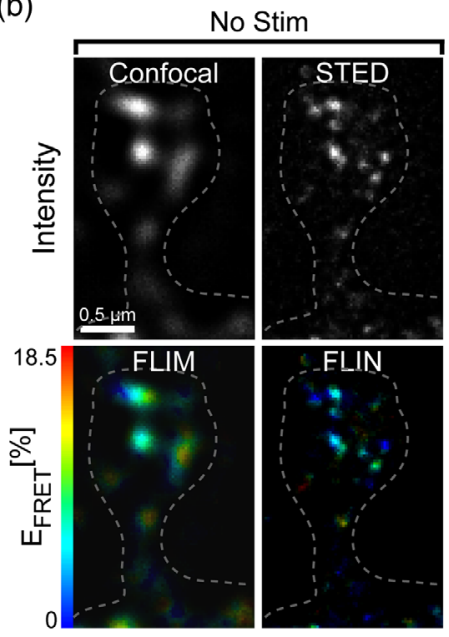

cLTP

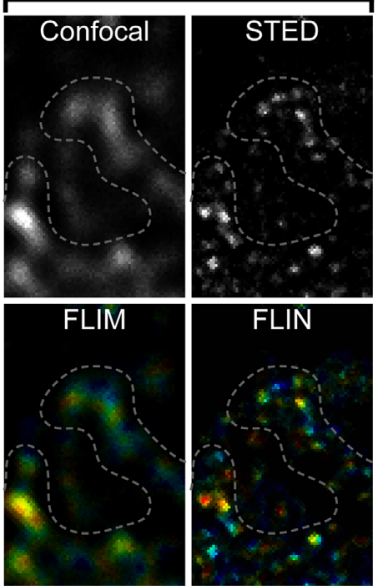

Fig. 6 Assessment of CaMKII interaction with NMDA receptor nanoclusters in dendritic spines. (a) Double immunolabeling configuration of $\alpha$ CaMKII with mouse anti- $\alpha$ CaMKII/GaM-ATTO 647N and rabbit anti-GluN2B/GaR-ATTO 594 (top cartoon). (b) Confocal and STED images of dendritic spines (top images) and corresponding intensity weighted color-coded images of FRET efficiencies (bottom images) shown with FLIM and FLIN, in the indicated conditions (preincubated in AP5 or after cLTP stimulation). (c) The distribution of FRET efficiencies over all pixels indicates a wider distribution of lifetimes in FLIN compared to FLIM. (d) The median FRET efficiencies per spine cluster was significantly higher in $\operatorname{cLTP}(7.5 \%, I Q R=8.9 \%, n=219$ clusters, four neurons), compared to the AP5 condition $(6.1 \%$, $\mathrm{IQR}=8.0 \%, n=320$ clusters, eight neurons) $(p=0.04)$.

C-terminus (ATTO 594-Donor) and $\alpha$ CaMKII (ATTO $647 \mathrm{~N}$ Acceptor) with a mouse antibody [Fig. 6(a)]. With FLIN, but not with FLIM, we could measure variable degrees of FRET in resolved nanoclusters inside a single spine [Fig. 6(b)]. This is characterized by a wider distribution of $E_{\mathrm{FRET}}$ for the FLIN images [Fig. 6(c), STD FLIM $8.61 \%$ and STD $_{\text {FLIN }}$ $10.01 \%]$, which can be explained by fluorescence lifetime averaging in FLIM due to lower resolution. Under these conditions, the fluorescence lifetime was significantly lower to that of the donor alone (Sec. 5.3 and Fig. 12), suggesting basal binding of CaMKII to GluN2B. In dendritic spines exposed to a cLTP stimulus, we observed a $15 \%$ increase of FRET efficiency in GluN2B nanoclusters compared with dendritic spines incubated in AP5 [Fig. 6(d)]. These results indicate that our FRET-FLIN method can provide subspine distribution of interactions between a synaptic receptor and a binding partner.

\subsection{Association of AMPA Receptors with Stargazin}

As a final test case, we examined the association of stargazin with the AMPA receptor on the surface membrane of dendritic spines. The trafficking of AMPA receptors to synapses has been shown to critically depend on its auxiliary subunit stargazin. ${ }^{38-42}$ Interestingly, recent evidence indicated that AMPA receptors may dissociate from stargazin to exit the synapse. ${ }^{43} \mathrm{We}$, thus, aimed to label surface AMPAR and stargazin to examine whether their interaction varies across different compartments on the membrane. We cotransfected neurons with GFP-GluA1 and HA-stargazin and performed immunolabeling in nonpermeabilized neurons to reveal only surface receptors, using anti-GFP (ATTO 594) and anti-HA (ATTO 647N) [Fig. 7(a)]. STED nanoscopy revealed resolvable clusters of GFP-GluA1 throughout the dendritic membranes [Fig. 7(c)]. ${ }^{4-46}$ Figure 7(b) shows the confocal image from HA-stargazin (red) overlaid with the STED image of GFP-GluA1 (green). FLIN revealed that a significant fraction of GFP-GluA1 nanoclusters exhibited some degree of interaction with HA-stargazin (Sec. 5.3 and Fig. 12). Meanwhile, FRET-FLIN analysis revealed a higher levels of FRET on dendritic spine membrane compared with the dendritic shaft [Figs. 7(e) and 7(f)]. These results suggest that extrasynaptic AMPA receptors are less associated with stargazin, compared to synaptic ones. ${ }^{43}$

\section{Discussion}

We demonstrate here the possibility to resolve FRET interactions inside nanoscale protein clusters in fixed neurons, using immunocytochemistry, STED nanoscopy, and fluorescence lifetime imaging. This demonstration was possible by the successful combination of STED and FLIM applied to FRET measurements. For cellular structures as small as dendritic spines, the capacity to resolve local protein interactions is essential to understand synaptic function.

While FRET provides information on protein interaction, when measured with confocal microscopy, it lacks the spatial resolution to provide information on the precise nanodomain where the interaction occurs. Combining STED with FRET overcomes this limitation. The combination of super-resolution with FRET has, thus far, been very limited. Gorlitz et al. developed a method in which they overlaid diffraction-limited FRETFLIM images, collected with time-gated wide-field imaging, on structured illumination intensity images of $~ 120$-nm lateral resolution. Winkler et al. $^{6}$ used a single-molecule imaging method (universal-point-accumulation-in-the-nanoscale-topography or uPAINT) to evaluate epidermal growth factor receptor dimerization. The labeling method used in uPAINT limits FRET measurements to plasma membrane proteins.

STED and FLIM have already been combined to facilitate multicolor imaging or to monitor the depletion process..$^{10,25,47}$ FLIM has been shown to be a robust approach to quantify 
(a)

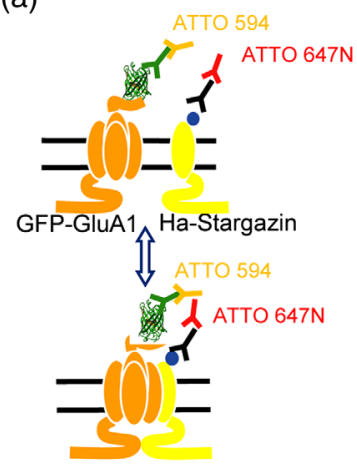

(d)

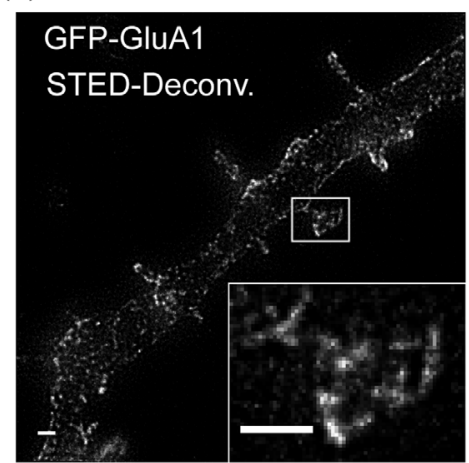

(b)

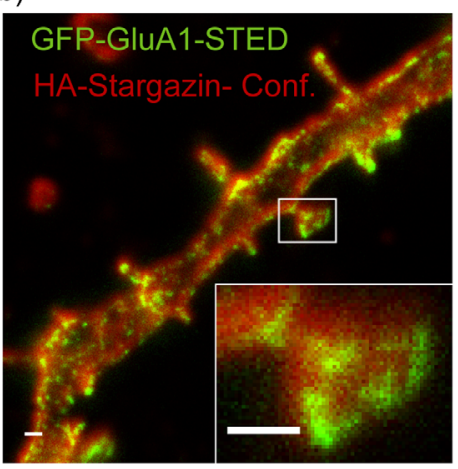

(e) (c)

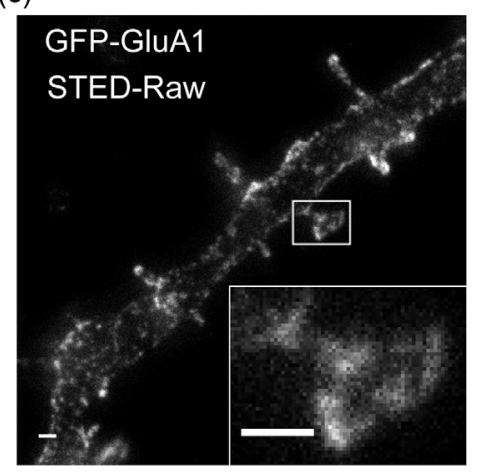

(f)
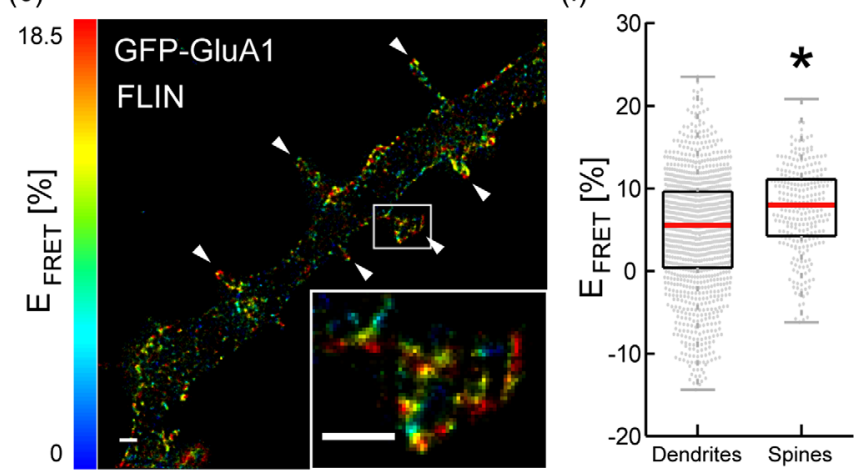

Fig. 7 Levels of association between AMPA receptors and stargazin in spines and dendrites. (a) Double immunolabeling configuration of GFP-GluA1 with mouse anti-GFP/GaM-ATTO 594 (Donor) and HAstargazin with rat anti-HA/GaR-ATTO 647N (Acceptor). (b) STED image of GFP-GluA1 (Donor) and confocal (Conf.) image of HA-Stargazin (Acceptor) on a transfected dendrite and an inset showing a dendritic spine. (c) STED raw intensity image of the donor showing GluA1 nanoclusters in spines and dendrites. (d) Corresponding deconvolved image of that showed in (c) (Richardson-Lucy deconvolution, simulated PSF of $60 \mathrm{~nm}$ FWHM). (e) Intensity-weighted FLIN image of the deconvolved image in (d) depicting higher FRET level in spines (white arrows) compared to dendrites. Inset: crop of one spine showing nanoclusters of fluorescently labeled AMPARs exhibiting different levels of FRET with fluorescently labeled stargazin. (f) The median FRET efficiencies per nanocluster on the membrane surface was significantly higher in spines $(8.0 \%, \mathrm{IQR}=6.9 \%, n=271$ clusters, 10 neurons) compared with dendrites $(5.5 \%, \mathrm{IQR}=8.0 \%, n=1058$ clusters, 10 neurons $)\left(p=9.45 \times 10^{-10}\right)$. Scale bars $500 \mathrm{~nm}$, inset: $1.56 \times 1.18 \mu \mathrm{m}$.

FRET. $^{48,49}$ The combination of STED and FLIM to measure FRET becomes a complementary approach to investigate molecular interactions inside nanoclusters of intracellular proteins.

For our demonstration, we performed FLIN on fixed samples of cultured neurons, enabling sufficient photon collection from nanodomains. Fixation with and without specific treatments can be used to assess changes in FRET as a proxy of signaling events at nanometric resolution. We targeted proteins that participate in synaptic signaling and plasticity in dendritic spines, for which specific antibodies or tagged recombinant versions were available. As the effectiveness and selectivity of antibodies is highly critical, we chose in some cases to transfect recombinantly tagged proteins, for which we had access to reliable antibodies. We used primary and secondary fluorescent antibodies, which introduce additional spacing and fluorophore orientation considerations, increasing uncertainties on the distance between epitopes. Despite these limitations, immuno-FRET has been used for assessing protein interactions. ${ }^{50,51} \mathrm{We}$ were able to use this approach to measure differential GluA1 interactions with stargazin in distinct membrane regions. Furthermore, our measurements made with NMDA receptors tagged with epitopes at different locations confirmed that the method bares sensitivity to discriminate distances within the FRET range on the same protein complex. An increase in FRET between two separately labeled proteins level might reflect an increased fraction of pairs interacting but might also reflect a conformational change in one or both of the interacting proteins that bring the labeled tags closer.

To reduce the uncertainty introduced by antibody labeling, pairs of fluorescent proteins could be used as tags for FRET-FLIM. ${ }^{18,23,52-54}$ However, STED-based FLIN with fluorescent proteins would introduce significant photobleaching due to their lower photostability compared with organic dyes. ${ }^{55,56}$ Furthermore, for each possible pair of fluorescent protein, a detailed characterization of the impact of the depletion pulse on the acceptor fluorescent protein would need to be performed.

Our simulations confirmed that the choice of analysis method of fluorescence lifetime impacts significantly on the results when photon collection is limited. This is particularly relevant when making measurements from nanoscalesized regions. The LS method is not optimal with limiting photon counts, making the MLE and MT methods preferable. 
Although the MT method provides fluorescence lifetime values with the least variations, it introduces an offset when FRET is present, underestimating higher levels of FRET, as predicted by our simulation and confirmed with experimental data (Sec. 5).

Our observation of GluN2B nanoclusters exhibiting interactions with CaMKII even before activating NMDA receptors with a cLTP stimulus may seem surprising. However, we found that GluN2B binding to CaMKII is highly persistent, even in low calcium condition. ${ }^{36,37}$ Thus, CaMKII may remain stably bound to GluN2B, from prior spontaneous activity of the neurons. This is also consistent with a basal level of autophosphorylated CaMKII that we observed in the spines. ${ }^{57}$ The activity-dependent increase in FRET that we observed between CaMKII and GluN2B, despite being small, is consistent with observations of activity-dependent translocation of GFP-CaMKII to dendritic spines using time-lapse imaging. ${ }^{28,32,36,37,58}$ In those studies, GFP-CaMKII was also shown to be somewhat enriched in dendritic spines prior to NMDA receptor activation. We proposed that prebound CaMKII may actually serve as the binding partner for additional recruitment of CaMKII to the PSD.$^{28}$ Indeed, we showed that the recruitment of CaMKII to dendritic spines can be supported by an activity-dependent self-association of the holoenzymes. ${ }^{28}$ Such added scaffold of CaMKII near the GluN2B c-tail, potentially forming "tower-like structures" extending away from the PSD ${ }^{59}$ may not produce more FRET between CaMKII and GluN2B, presumably because of the added distance between the additional stacked CaMKII holoenzymes and the GluN2B c-tail. This putative scenario could explain the limited increase we observed in FRET between CaMKII and GluN2B following stimulation.

The observation that GluA1 nanoclusters exhibiting different levels of interaction with stargazin on dendritic shaft vs spine membrane is quite interesting. Indeed, stargazin has been shown to promote immobilization of AMPA receptor in the PSD area, via binding to PSD95. ${ }^{30,41}$ Meanwhile, Constals et al. ${ }^{43}$ showed that AMPA receptor desensitization was associated with a decreased binding to stargazin, enabling the exit of receptors from the synapse. Our data are then consistent with the proposal that AMPA receptors near the PSD are preferentially associated with stargazin, compared with extrasynaptic receptors. The model of synaptic nanocolumns, describing receptor clusters in the PSD aligned with presynaptic release sites, would be strengthened by identifying binding partners responsible for the formation and plasticity of these nanocolumns. ${ }^{60}$ Our approach may be useful for such investigation.

Our results indicate that the combination of STED, FLIM, and immuno-FRET can reveal direct or indirect interactions between proteins within nanodomains, as exemplified by our measurements in dendritic spines. FRET-FLIN revealed different degrees of interactions between nanoclusters of proteins that are otherwise missed with conventional FRET-FLIM. The approach can be used to interrogate various pairs of interacting endogenous proteins, providing the availability of specific antibodies, or transfected tagged-proteins using well validated antitag antibodies. A valuable improvement in this method would be to add colors with STED resolution to detect additional proteins as landmarks. In the examples studied here, being able to locate the PSD or the presynaptic active zone would be very informative. Thus, this approach should help the investigation of cell signaling at the nanometer scale.

\section{Appendix A: FRET-FLIN Setup}

We built an STED microscope based on the work of Wildanger et al. ${ }^{13}$ and Bückers et al., ${ }^{10}$ (Fig. 8) using a single supercontinuum laser source (SC450-PP-He, 2MHZ, 4W, Fianium, NKT, Blokken, Denmark) to generate all excitation and depletion beams. The excitation and the depletion paths were splitted using a dichroic mirror (FF662-FDi01, Semrock, Rochester). The excitation beam was spectrally filtered with an acoustooptical-tunable-filter (AOTFnc-400.650-TN, AA optoelectronics, Orsay, France) and the output beam was spatially filtered through a polarization-maintaining single-mode fiber (PM460 HP, Thorlab, Newton) and the depletion path was additionally filtered with a pinhole. The $s$ and $p$ polarizations of the depletion beam were separated with a polarizing beam splitter. A vortex phase plate was positioned in each depletion path (VPP-1, RPC photonics, Rochester) to generate two donut-shaped depletion beams, for which the $\mathrm{s}$ and $\mathrm{p}$ polarizations had a clockwise or counter clockwise phase shift pattern, respectively. Both polarizations were then recombined on a common path. Two bandpass filters (FF01-720/13, Semrock) resulting in a Gaussian beam profile ranging from 713 to $726 \mathrm{~nm}$ were necessary to obtain a sharp spectral profile and therefore improve the intensity minima of the donut-shaped beam through uniform light retardation on the vortex phase plate. A half and a quarter wave plates were employed for fine tuning and circularization of the polarization. A delay line was used to adjust the timing between the excitation and the depletion pulses to $74 \mathrm{ps}^{61}$ The depletion and excitation beams were combined with a dichroic mirror (Z690sprdc-v2, Chroma Technology Corp., Bellows Falls, USA) and the fluorescence was separated with a custom dichroic mirror (Custom STED polychroic V6-246389, Chroma Technology Corp., Bellows Falls). The scanning unit on the microscope (Zeiss Axio Examiner) was built to minimize aberrations at the focal spot and optimize the depletion donut pattern. For this purpose, two galvanometric mirrors $(6215 \mathrm{H}$, from Cambridge technology) were placed in the conjugated plane of the back aperture of the microscope objective (Plan Apochromat, 63X, 1.4NA, Zeiss) in a $4 \mathrm{f}$ configuration.

The detected photons were splitted with a 660 single-edge dichroic (FF660-Di02, Semrock) to discriminate between the donor (ATTO 594) and the acceptor (ATTO 647N) (Fig. 9). The FLIM/FLIN signal of the donor was acquired with a TCSPC card (SPC150, Becker \& Hickl, Berlin, Germany) on a fast photomultiplier tube (PMT) (PMC-100-20, Boston electronics, Brookline, USA) with a FF01-609/57 (Semrock) detection filter (Fig. 9). For the acceptor, the intensity signal was acquired on a data acquisition board (NiDAQ, PCI-6111, National Instrument) using a custom integration system with a regular PMT (R3896, and high-voltage socket with transimpedance amplifier C7950, Hamamatsu, Japan) and a FF01-685/40 (Semrock) detection filter. For both emission paths, a 150-mm detection lens was combined to a $75-\mu \mathrm{m}$ pinhole in front of the PMT.

Prior to each imaging session, the proper alignment of STED donuts over the excitation beam was tested in reflectance with 100 -nm gold beads. The acquisition was performed using a modified version of the open-source software ScanImage $3.8^{62}$ in addition to the TCPSC acquisition software (SPCM v9.6., Becker \& Hickle). To collect $>1000$ photons in FLIN mode, a total of 20 images (45 s per image) were recorded for an overall acquisition time of $\sim 15 \mathrm{~min}$. Postprocessing 


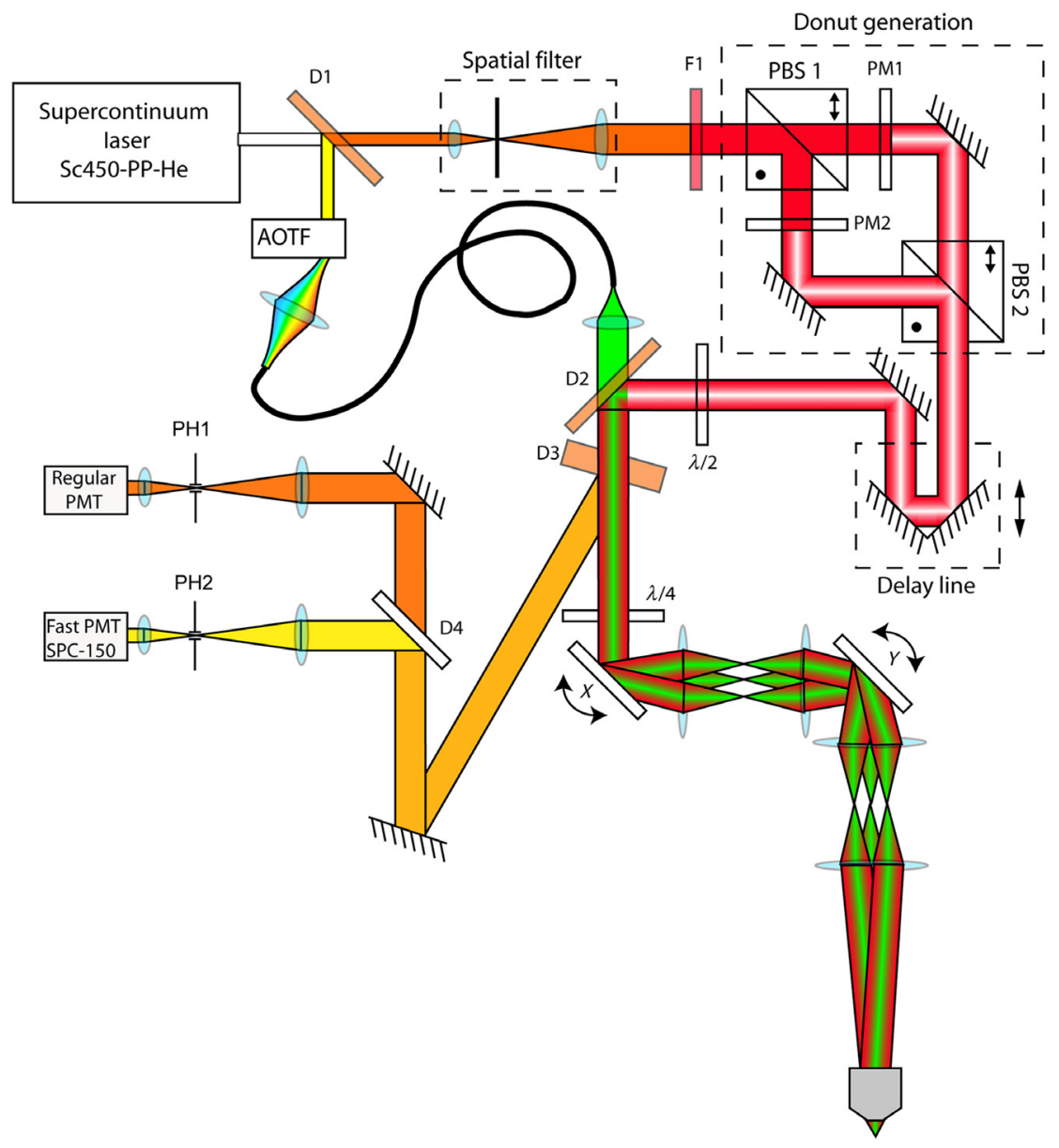

Fig. 8 FRET-FLIN setup acronyms: dichroic mirrors (D1, D2, D3, D4), acousto-optical-tunable-filter (AOTF), band pass filters (F1), polarizing beam splitters (PBS1, PBS2), phase masks (PM1, PM2), galvanometric mirrors $(X, Y)$, half waveplate $(\lambda / 2)$, quarter waveplate $(\lambda / 4)$, confocal pinholes (PH1, $\mathrm{PH} 2)$, photomultiplier tubes (fast PMT with SPC-150-TCSPC electronics for the donor fluorescence and regular PMT for the acceptor fluorescence).

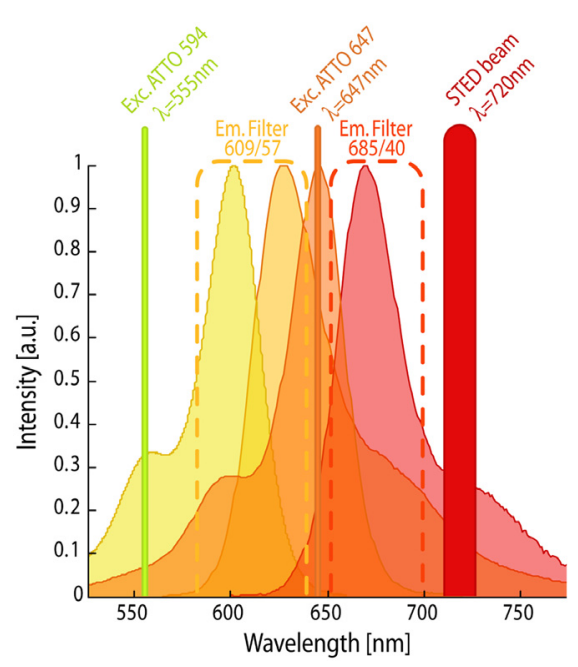

Fig. 9 Fluorophore, laser, and filter configuration for one color FRETFLIN imaging. The emission spectrum of ATTO 594 overlaps with the excitation spectrum of ATTO $647 \mathrm{~N}$ enabling FRET (donor/acceptor $R_{0}$ value of $7.4 \mathrm{~nm}$ ) (Spectra from www.atto-tec.com). The excitation wavelengths were selected on the AOTFs at $555 \mathrm{~nm}$ (for ATTO 594) and $647 \mathrm{~nm}$ (for ATTO 647N) and the emission band-pass filters were $609 / 57 \mathrm{~nm}$ and $685 / 40 \mathrm{~nm}$, respectively. The STED beam wavelength was centered at $720 \mathrm{~nm}$ with a bandwidth of $13 \mathrm{~nm}$. realignment using a normalized two-dimensional (2-D) cross correlation was performed to correct for sample drift. The realigned images were summed, resulting in a single frame of $12.3 \times 12.3 \mu \mathrm{m}(512 \times 512$ pixels of $24 \mathrm{~nm})$ for the analysis.

The resolution of the STED microscope was characterized by measuring the FWHM on single clusters of the neuronal glutamate receptor GluN2B and antibody clusters on a glass coverslip. For GluN2B in dendrites, a mean FWHM of $73.3 \mathrm{~nm}$ (STD $13.7 \mathrm{~nm}$ ) was obtained while for antibody clusters it was $66.9 \mathrm{~nm}$ (STD $11.0 \mathrm{~nm})$ (Fig. 10).

\section{Appendix B: Simulations and Image Analysis}

\subsection{Simulations}

We have based our simulations on a simplified transfer equation of fluorescence and stimulated emission to model emission and depletion processes involved in FLIN [Eq. (1), adapted from Siegman $^{63}$ and Lakowicz et al. ${ }^{48}$ ]:

$$
\frac{\partial N 1}{\partial t}=N_{0} k_{\mathrm{exc}} h_{\mathrm{exc}}-N_{1}\left(k_{\mathrm{fluo}}+k_{\mathrm{FRET}}+k_{\mathrm{STED}} h_{\mathrm{STED}}\right)
$$


(a)

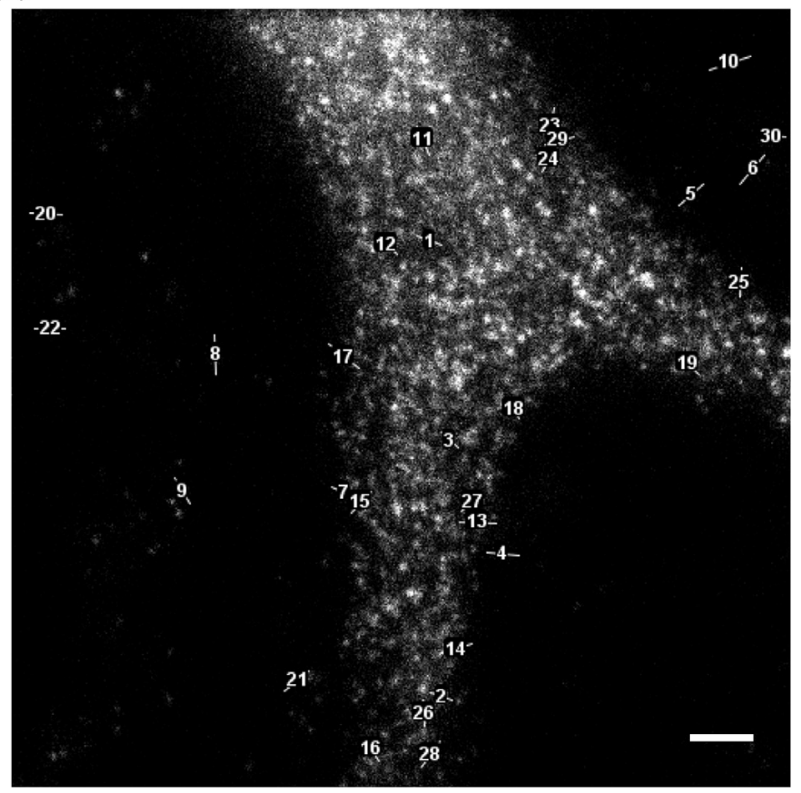

(b)

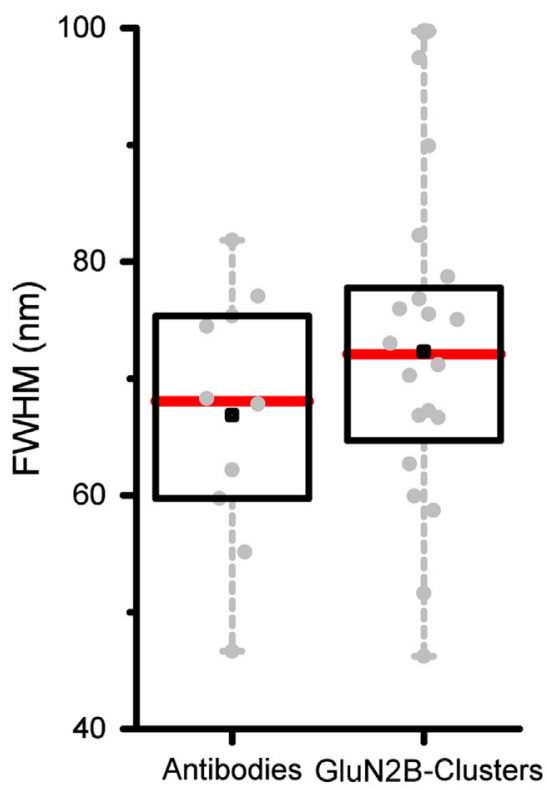

(c)
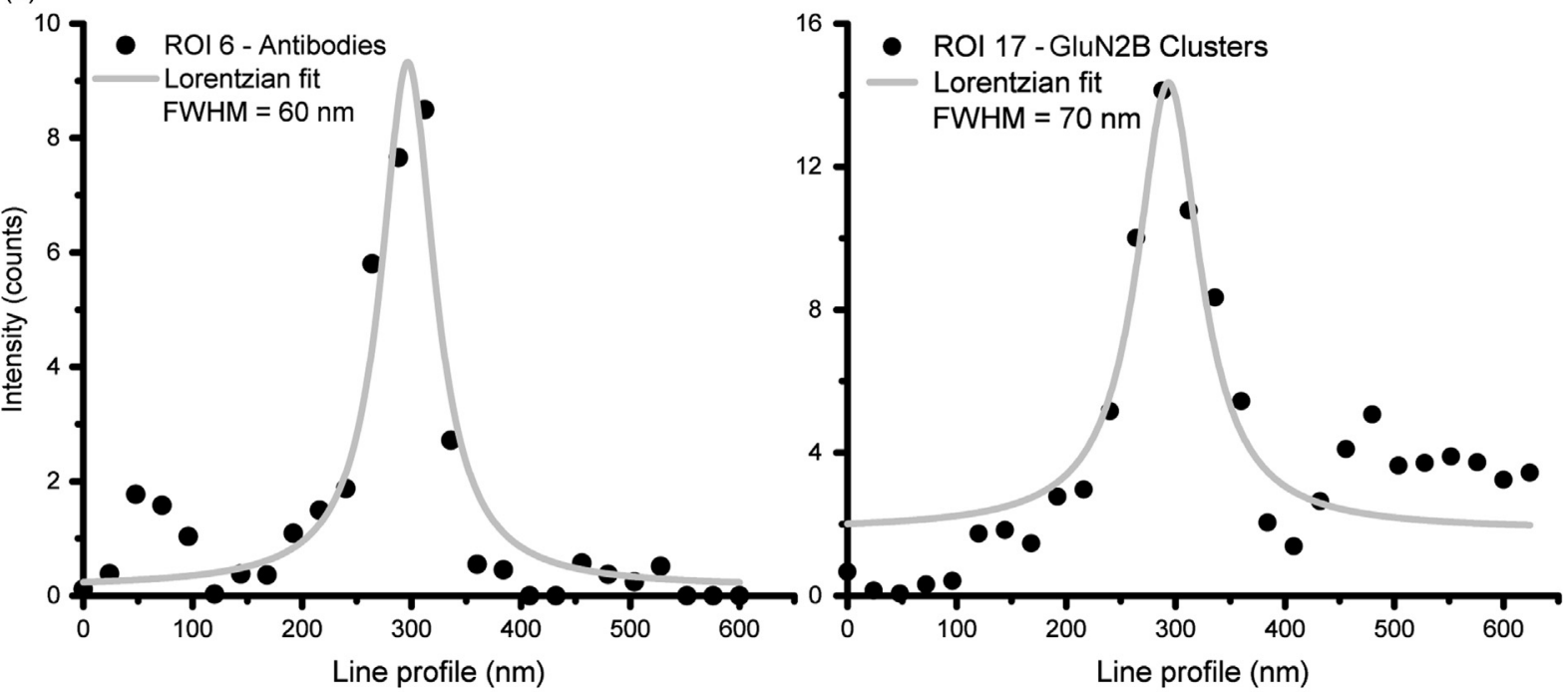

Fig. 10 Resolution performance of the custom STED system on immunolabeled GluN2B in fixed cultured hippocampal neurons. (a) STED imaging of GluN2B labeled with mouse anti-GluN2B and GAM-ATTO 594 (raw data). Line profiles (white lines) were measured on unspecific single antibody-nanoclusters (outside of the neuron) for resolution estimation, and on GluN2B-nanoclusters. (b) Measured FWHM of a Lorentzian curve fit on the line profiles showed in (a) for both single antibodies $(66.9 \pm 11.0 \mathrm{~nm}$, $n=10)$ and GluN2B $(73.3 \pm 13.7 \mathrm{~nm}, n=20)$ nanoclusters (mean $\pm \mathrm{SD}$, both population are normally distributed, Shapiro-Wilk Normality Test). (c) Representative fits of the fitted line profiles for antibody (left) and GluN2B (right) nanoclusters.

where

$h_{\mathrm{exc}}=e^{\left[-\frac{\left(t-d_{\mathrm{exc}}\right)^{2}}{c_{\mathrm{exc}}}\right]}$,

$k_{\mathrm{exc}}=I_{\mathrm{exc}} \sigma_{\mathrm{exc}}$,

$k_{\text {fluo }}=\frac{1}{\tau_{\text {fluo }}}$,

$k_{\text {FRET }}=k_{\text {fluo }}\left(\frac{R_{0}}{r}\right)^{6}$,
$h_{\mathrm{STED}}=e^{\left[-\frac{\left(t-d_{\mathrm{STED}}\right)^{2}}{c_{\mathrm{STED}}}\right]}$,

$k_{\mathrm{STED}}=I_{\mathrm{STED}} \sigma_{\mathrm{STED}}$,

where $N_{1}$ and $N_{0}$ represent the number of electrons in the excited state and ground state of a molecule, respectively. The rate constants for the excitation, fluorescence emission, stimulated emission, and FRET are indicated with $k_{\text {exc }}, k_{\text {fluo }}$ (inverse of fluorescence lifetime $\tau_{\text {fluo }}$ ), $k_{\mathrm{SE}}$, and $k_{\mathrm{FRET}}$, respectively. The depletion efficiency is related to the intensity $\left(I_{\text {STED }}\right)$ and the temporal Gaussian profile $\left(h_{\mathrm{STED}}\right)$ of the depletion beam 
Table 1 Simulation parameters of FLIN experiments.

\begin{tabular}{lccc}
\hline & Excitation beam & STED beam & Fluorescent molecule \\
\hline Pulse FWHM & $200 \mathrm{ps}$ & $200 \mathrm{ps}$ & - \\
Focal spot FWHM & Gaussian $250 \mathrm{~nm}$ & Donut $432 \mathrm{~nm}$ peak to peak & - \\
Wavelength & $555 \mathrm{~nm}$ & $720 \mathrm{~nm}$ & - \\
Laser intensity & $100 \mathrm{~W} / \mathrm{cm}^{2}$ & $100 \mathrm{MW} / \mathrm{cm}^{2}$ & $3.5 \times 10^{-16} \mathrm{~cm}^{2}$ \\
Delay & $1 \mathrm{~ns}$ & $1.074 \mathrm{~ns}$ & $3.5 \times 10^{-16} \mathrm{~cm}^{2}$ \\
Absorption cross section & - & - & - \\
STED cross section & - & - & $3.2 \mathrm{~ns}$ \\
Lifetime & - & - & $50 \%$ \\
Maximum FRET efficiency & - & - & $7.4 \mathrm{~nm}$ \\
$R_{0}$ & & & \\
\hline
\end{tabular}

as well as the stimulated emission cross section $\left(\sigma_{\mathrm{STED}}\right) . R_{0}$ represents the Förster radius (radius for which the FRET efficiency equals 50\%) and $r$ is the distance between donor and acceptor. Using a numerical solution of that equation, we were able to evaluate the distribution of fluorescence lifetime emitted by a molecule. The properties used to simulate the fluorescence, the excitation, and the depletion are listed in Table 1.

To simulate a case of interacting pairs of molecules, a FRET efficiency of $50 \%$ ( $r=R_{0}=7.4 \mathrm{~nm}$ between ATTO 594 and ATTO $647 \mathrm{~N}$ ) and a FRET probability of $30 \%$ between the two proteins were assumed. This corresponds to a measured FRET efficiency of $15 \%$. The lifetime of the donor alone was set to 3.2 ns. Consequently, the calculated lifetime for $15 \%$ FRET efficiency was $2.7 \mathrm{~ns}$. The double exponential decay was evaluated by the rate equations with a $30 \%$ probability to obtain FRET (70\% probability to measure the donor lifetime without FRET interaction) for each simulated molecule.

FLIM and FLIN images were simulated [Figs. 1(a)-1(e)] by randomly positioning 250 molecules inside a dendritic spine shape $(2.8 \times 2.0 \mu \mathrm{m})$. A Gaussian excitation PSF and a sinusoidal donut depletion PSF were simulated using the numerical solution of the transfer Eq. (1). The effective fluorescence PSF was applied on each pixel containing a simulated molecule. Subsequently, all photons with shot noise (using Poisson random generator) were added up for each pixel.

The performance of three FLIM analysis methods (LS, MT, and MLE) was compared using simulated lifetime histograms with variable FRET levels. A total of 100 independent simulations were performed with photon counts ranging from 100 to 10,000. The simulations were averaged per photon count and compared with the ground truth.

\subsection{Curve Fitting and Lifetime Quantification}

For each image or simulation, the lifetime was evaluated using the indicated algorithm (LS, MLE, or MT). Minimization algorithms were based on the following model:

$f(t)=N_{\text {photons }}\left[e^{\left(\frac{-t}{\tau_{m}}\right)} * \operatorname{IRF}\right]$, where $N_{\text {photons }}$ is the total number of collected photons and $\tau_{m}$ is the average fitted lifetime. The measured instrument response (IRF) was convolved with the fluorescence exponential decay. The acquisition time resolution was set to $40 \mathrm{ps}$ and a temporal binning of 4 was used, leading to a time resolution of 158 ps. A convolutive spatial binning of 2 was applied, corresponding to an effective pixel size of $48 \mathrm{~nm}$ for the FLIN signal. The photons outside of the fitting window (red and green dashed lines in Fig. 11) were excluded from the fitting routine. The fitting algorithms were based on the MATLAB (Mathworks) function fmincon, which finds the minimum of constrained multivariable nonlinear function. Cost function for minimization was based on the work of Maus et al. ${ }^{21}$ for both MLE [Eq. (3) and LS Eq. (4)]:

$$
\begin{aligned}
& \operatorname{MLE}_{\text {cost function }}=\left[\frac{2}{(k+\nu)}\right] \sum_{0}^{t} I(t) \log \left[\frac{I(t)}{f(t)}\right] \\
& \mathrm{LS}_{\text {cost function }}=\left[\frac{1}{(k+\nu)}\right] \sum_{0}^{t} \frac{[I(t)-f(t)]^{2}}{I(t)},
\end{aligned}
$$

where $k$ is the number of data points, $\nu$ is the number of parameters, $I(t)$ is the measured data, and $f(t)$ is the function to be fitted [Eq. (2)]. In the case of the MT method, ${ }^{22}$ we used the photon mean arrival of the IRF and subtracted it from the data itself: ${ }^{19,22}$

$$
\tau_{\mathrm{MT}}=\frac{\sum_{0}^{t}[t \cdot I(t)]}{\sum_{0}^{t} I(t)}-\frac{\sum_{0}^{t}[t \cdot \operatorname{IRF}(t)]}{\sum_{0}^{t} \operatorname{IRF}(t)}
$$

Note here that the MT method does not provide an absolute lifetime value. To correct for that bias in single-exponential lifetime, it is necessary to evaluate the lifetime of the donor only using a fitting approach on a known sample without acceptor. The obtained correction factor can be applied on all experiment made in that condition. 

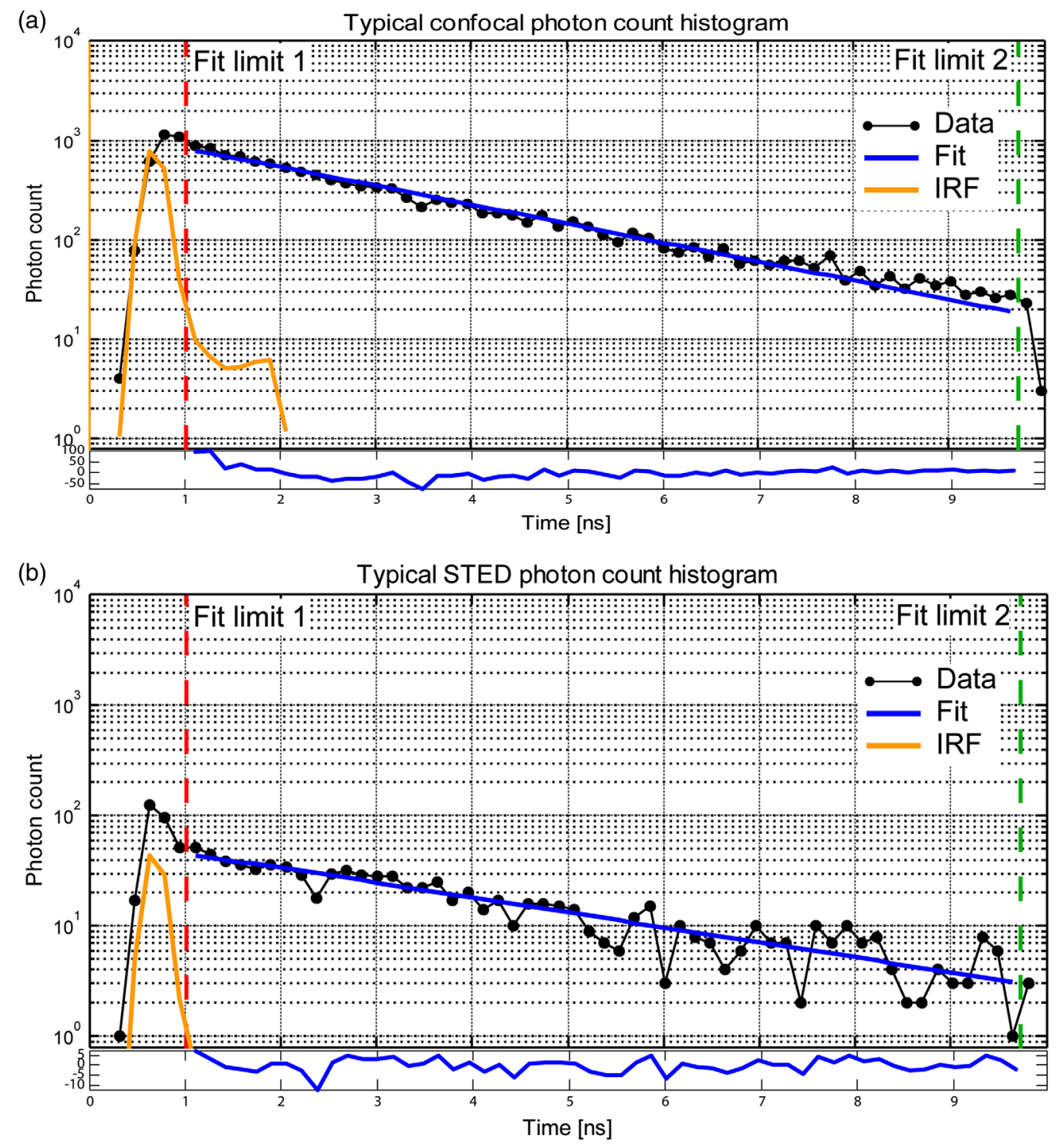

Fig. 11 Photon count histogram. (a) Distribution of photons over time for a typical FLIM measurement on a dendritic spine. The exponential function fit (blue line) was convolved with the IRF; the time window limits for the fit are indicated by the red and green dashed lines. The photon count of this representative histogram was 14,245 photons. (b) Photon distribution of the same pixel as in A under FLIN measurement. Data between the red and green dashed lines were used for fitting analysis (after the STED pulse and the peak signal produced by the fluorophores before begin of STED effect). The photon count in this histogram was 1044 photons.

\subsection{Image Analysis}

The quantification of fluorescence lifetime was performed as described in the previous section (curve fitting and lifetime quantification) using the MLE algorithm for curve fitting. We applied Richardson-Lucy deconvolution on the intensity signal (2-D Gaussian function for confocal and 2-D Lorentz function for STED images) using a build-in MATLAB function deconvlucy.m and emulated PSFs of 270 and $60 \mathrm{~nm}$ for confocal and STED images, respectively. To measure the individual cluster intensities and the associated lifetime values, clusters detected using an adaptive threshold algorithm combined with a morphological analysis. ${ }^{64}$ Those segmented regions were associated with the lifetime image and the lifetime values for each cluster were retrieved by averaging all pixel inside a given cluster. A colormap image was obtained from the determined lifetime values for each pixel and multiplied by the corresponding intensity image to generate the intensity weighted lifetime images. For spine area selection, regions were manually drawn around excrescences on dendrites.

For each experiments, a control immunolabeling with the donor alone was performed to determine the donor lifetime under the same biological and imaging conditions (Fig 12). All pixels of the donor-alone control images were averaged to evaluate the value of donor lifetime. The FRET efficiency was calculated with $E_{\mathrm{FRET}}=1-\left(\tau_{D+A} / \tau_{D}\right)$, as $\tau_{D}$ represents the measured fluorescence lifetime of the donor alone and $\tau_{D+A}$ is the measured fluorescence lifetime of the donor in presence of acceptor. Note that the measured FRET efficiency values can be negative due to the intrinsic distribution of the fluorescent molecule lifetime. 


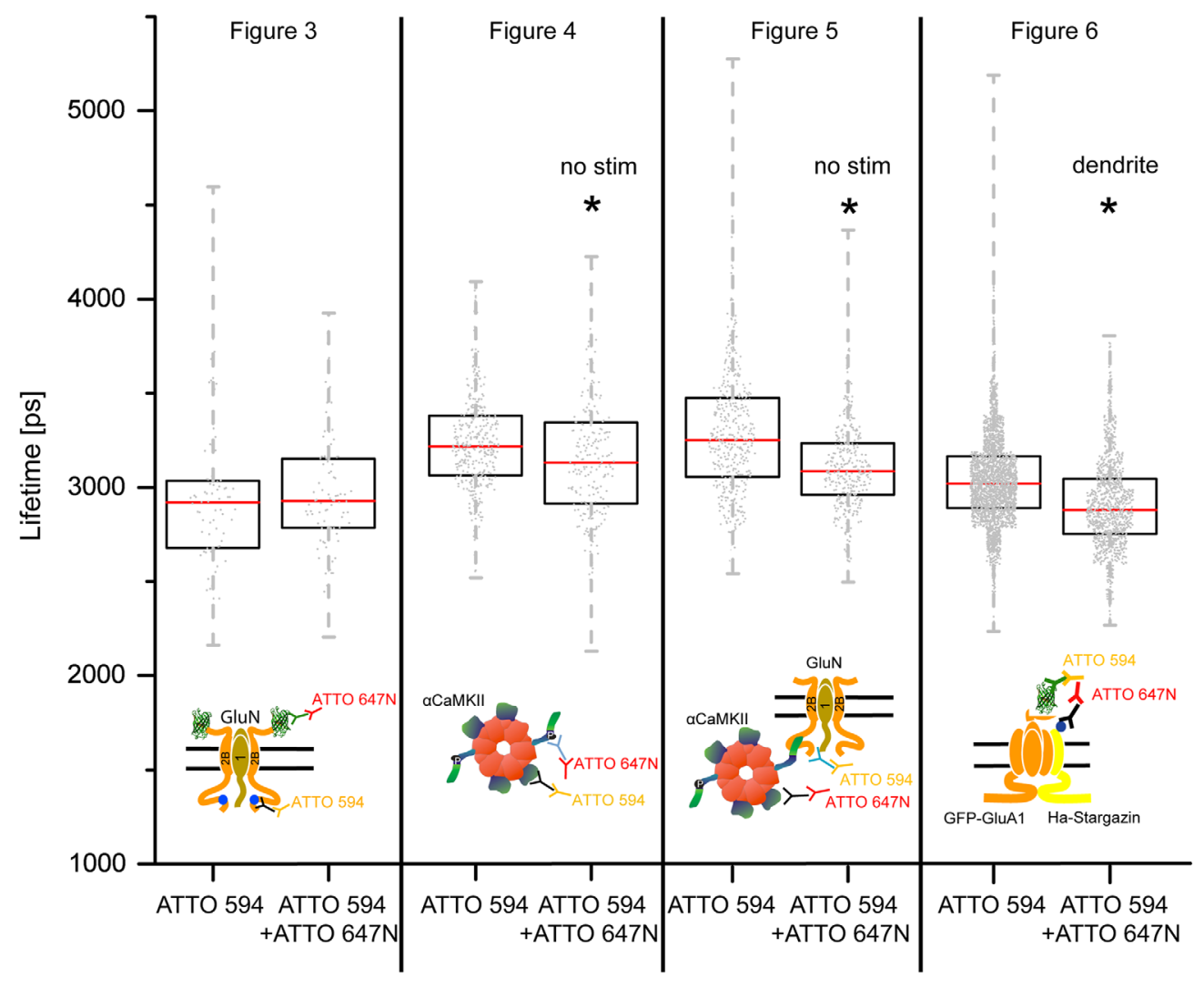

Fig. 12 Comparison of FLIN between donor alone and donor-acceptor for experiments shown in Figs. 4-7 (Fig. 4 panel). The fluorescence lifetime of ATTO 594 alone, labeling HA in GFP-GluN2B-HA, is not significantly different to that in the presence of the acceptor ATTO $647 \mathrm{~N}(p=0.3282)$ (Fig. 5 panel). The fluorescence lifetime of ATTO 594 alone, labeling $\alpha$ CaMKII, is significantly higher than in the presence of the acceptor Atto647 ( $p=0.00077)$ (Fig. 6 panel). The fluorescence lifetime of ATTO 594 alone, labeling GluN2B, is significantly higher than in the presence of the acceptor ATTO $647 \mathrm{~N}\left(p<2.2 \times 10^{-16}\right)$ (Fig. 7 panel). The fluorescence lifetime of ATTO 594 alone, labeling GFP-GluA1, is significantly higher than in the presence of the acceptor ATTO $647 \mathrm{~N}$ (on stargazin) $\left(p<2.2 \times 10^{-16}\right)$.

\subsection{Statistical Analysis}

Statistical analyses were performed over cluster distributions. Outliers were defined as values larger than $q_{3}+1.5\left(q_{3}-q_{1}\right)$ or smaller than $q_{1}-1.5\left(q_{3}-q_{1}\right)$, where $q_{1}$ and $q_{3}$ are the 25 th and 75 th percentiles, respectively. The non-normal distributed datasets were tested by a Wilcoxon rank sum test and statistical significance was determined with $p \leq 0.05$ using twotailed tests. All data are presented as median with IQR value. Data were analyzed using MATLAB statistical toolbox.

\section{Appendix C: Material and Methods}

\subsection{Neuronal Cultures and Transfection}

Dissociated hippocampal neurons were prepared as described. ${ }^{28,65}$ Before dissection of hippocampi, neonatal rats were sacrificed by decapitation, in accordance with the procedures approved by the animal care committee of Université Laval. Neurons were transfected with the plasmids encoding GFP-GluN2B-HA, GluN1-GFP, GluN2B-HA, SEP-GluA1, or HA-Stargazin at 11-14 DIV using Lipofectamine 2000 (Invitrogen) as described previously. ${ }^{66}$ Fixation was performed $24 \mathrm{~h}$ after transfection. To reduce toxicity generated by the overexpression of the plasmids GFP-GluN2B-HA, GluN1-GFP, and GluN2B-HA, $200 \mu \mathrm{M}$ AP5 (Cayman) was added $3 \mathrm{~h}$ after transfection.

The plasmid GFP-GluN2B-HA was generated by PCR amplification of the cDNA encoding GFP-GluN2B with primers 5'-CAAGACACGTGCTGAAGTCAAG-3' and 5'GCTAGTGGTCCACATGTAGTACCG-3'. The PCR product was then digested with SnaBI-XhoI (digestion product contains incomplete CMV promoter, first part of GluN2B and the GFP) and inserted into the SnaBI-XhoI site of GluN2B-HA. GluN2BHA was generated by inserting a HA tag at amino acid 1275 of GluN2B in a pRK5 vector. SEP-GluA1, HA-Stargazin, and GluN1-GFP were described previously. ${ }^{18,30,32}$

\subsection{Immunocytochemistry}

Cells were fixed either in methanol $\left(-20^{\circ} \mathrm{C}\right)$ or in a freshly prepared $4 \%$ paraformaldehyde (PFA) solution (4\% sucrose, 100 $\mathrm{mM}$ phosphate, $2 \mathrm{mM}$ NaEGTA) [room temperature (RT)] for $10 \mathrm{~min}$. PFA fixation was used only for the GluA1Stargazin experiment to label exclusively extracellular membrane proteins. After fixation, cells were washed three times for $5 \mathrm{~min}$ in PBS (PBS with $0.1 \mathrm{mM}$ Glycine for PFA fixation). To limit unspecific binding, cells were first incubated for $1 \mathrm{~h}$ in a blocking solution (BS) consisting of PBS completed with 10\% normal goat serum. Primary antibody incubation was performed 
with BS for $2 \mathrm{~h}$ at RT or overnight at $4^{\circ} \mathrm{C}$. After five washes in PBS, the secondary antibodies were applied with BS for $1 \mathrm{~h}$ at RT.

The following antibodies were used: rabbit antiphosphoT286CaMKII (1:500, Cell signaling technology), Mouse anti- $\alpha$ CaMKII (1:200, ThermoFisher Scientific), Rabbit anti-GluN2B-CT (1:500, Alomone, AGC-003), Mouse anti-GFP (1:500, ThermoFisher Scientific), Rat anti-HA (1:250, Roche), and their corresponding secondary antibodies, ATTO $594(1: 500)$ or ATTO $647 \mathrm{~N}$ (1:500, ATTO-TEC).

To measure the influence of cLTP stimulation on CaMKII T286 autophosphorylation, neurons were incubated for $5 \mathrm{~min}$ in free-magnesium heated artificial cerebrospinal fluid $\left(0 \mathrm{Mg}^{2+}\right.$-ACSF) solution consisting of HBSS supplemented with, in mM: 10 HEPES, $1.2 \mathrm{Ca}^{2+}, 2$ glucose, 0.2 glycine, and 0.01 picrotoxin. Neurons were either fixed directly after stimulation (cLTP) or washed for $10 \mathrm{~min}$ in AP5 $(400 \mu \mathrm{M})$ containing regular ACSF (10 min post-cLTP).

The immunolabeled coverslips were mounted in 2,2'thiodiethanol (TDE, Sigma Aldrich), based on the protocol described in Staudt et al. ${ }^{67}$ This polymerization free mounting media minimizes lifetime alterations of fluorescent dyes and aberrations caused by refractive index $(n)$ mismatch $(n=1.5)$ (coverslips were incubated with gradually increasing concentration of TDE $(10 \%, 25 \%, 50 \%$, and $3 \times 97 \%)$ for $30 \mathrm{~min} /$ concentration to avoid cell shrinkage while completely removing water. $^{67}$

\section{Disclosures}

The authors declare no potential conflicts of interest with respect to the research, authorship, and/or publication of this article.

\section{Acknowledgments}

We thank F. Nault for preparing neuronal cultures, A. Castonguay and M. Lemieux for comments on the paper, the CERVO Molecular Tool Platform for cloning and preparation of plasmid DNA. We thank M. Stratton (UMass) for providing info on the structure of CaMKII. Research was funded by the Canadian Institute of Health Research (CIHR) and the Natural Science and Engineering Research Council of Canada (NSERC) (P.D.K.). C.T. was supported by a scholarship from the Fonds de Recherche du Québec Nature et Technologie; G.N. was supported by a CIHR studentship; S.L. and F.L.C. were supported by the Brain Canada Neurophotonics Platform and the NSERC training program in Biophotonics. C.T. built the FLIN setup, acquired all of the data and performed the simulations. C.T. and G.N. performed the immunocytochemistry. C.T., G.N., S.L., F.L.C., P.D.K. wrote the manuscript, made the figures, and performed the data analysis. C.T., D.C., and P.D.K. designed the study.

\section{References}

1. M. Elangovan, R. N. Day, and A. Periasamy, "Nanosecond fluorescence resonance energy transfer-fluorescence lifetime imaging microscopy to localize the protein interactions in a single living cell," J. Microsc. 205(1), 3-14 (2002).

2. W. Becker, "Fluorescence lifetime imaging-techniques and applications," J. Microsc. 247(2), 119-136 (2012).

3. S. Brasselet et al., "Single-molecule fluorescence resonant energy transfer in calcium concentration dependent cameleon," J. Phys. Chem. B 104(15), 3676-3682 (2000).
4. U. B. Choi et al., "Single-molecule FRET-derived model of the synaptotagmin 1-SNARE fusion complex," Nat. Struct. Mol. Biol. 17(3), 318-324 (2010).

5. K. Okamoto and Y. Sako, "Recent advances in FRET for the study of protein interactions and dynamics," Curr. Opin. Struct. Biol. 46, 16-23 (2017).

6. P. Winckler et al., "Identification and super-resolution imaging of ligand-activated receptor dimers in live cells," Sci. Rep. 3(1), 2387 (2013).

7. F. Görlitz et al., "Mapping molecular function to biological nanostructure: combining structured illumination microscopy with fluorescence lifetime imaging," in Optics in the Life Sciences Congress (2017).

8. S. W. Hell and J. Wichmann, "Breaking the diffraction resolution limit by stimulated emission: stimulated-emission-depletion fluorescence microscopy," Opt. Lett. 19(11), 780-782 (1994).

9. E. Auksorius et al., "Stimulated emission depletion microscopy with a supercontinuum source and fluorescence lifetime imaging," Opt. Lett. 33(2), 113-115 (2008).

10. J. Bückers et al., "Simultaneous multi-lifetime multi-color STED imaging for colocalization analyses," Opt. Express 19(4), 3130-3143 (2011).

11. M. D. Lesoine et al., "Supercontinuum stimulated emission depletion fluorescence lifetime imaging," J. Phys. Chem. B 116(27), 78217826 (2012).

12. I. Testa et al., "Dual channel RESOLFT nanoscopy by using fluorescent state kinetics," Nano Lett. 15(1), 103-106 (2015).

13. D. Wildanger et al., "STED microscopy with a supercontinuum laser source," Opt. Express 16(13), 9614-9621 (2008).

14. D. Wildanger et al., "A compact STED microscope providing 3D nanoscale resolution," J. Microsc. 236(1), 35-43 (2009).

15. W. Becker et al., "Fluorescence lifetime imaging by time-correlated single-photon counting," Microsc. Res. Tech. 63(1), 58-66 (2004).

16. F. Itri et al., "Identification of novel direct protein-protein interactions by irradiating living cells with femtosecond UV laser pulses," Biochem. Biophys. Res. Commun. 492(1), 67-73 (2017).

17. P. S. David Gerecht, M. A. Taylor, and J. Port, "Intracellular localization and interaction of mRNA binding proteins as detected by FRET," BMC Cell Biol. 11(1), 69 (2010).

18. K. Doré et al., "FRET-FLIM investigation of PSD95-NMDA receptor interaction in dendritic spines; control by calpain, CaMKII and Src family kinase," PLoS One 9(11), e112170 (2014).

19. W. Becker, The bh TCSPC Handbook, 7th ed., Becker \& Hickl GmbH, Berlin (2017).

20. T. A. Laurence and B. A. Chromy, "Efficient maximum likelihood estimator fitting of histograms," Nat. Methods 7(5), 338-339 (2010).

21. M. Maus et al., "An experimental comparison of the maximum likelihood estimation and nonlinear least-squares fluorescence lifetime analysis of single molecules," Anal. Chem. 73(9), 2078-2086 (2001).

22. R. Yasuda et al., "Supersensitive Ras activation in dendrites and spines revealed by two-photon fluorescence lifetime imaging," Nat. Neurosci. 9(2), 283-291 (2006).

23. S.-J. R. Lee et al., "Activation of CaMKII in single dendritic spines during long-term potentiation,” Nature 458(7236), 299-304 (2009).

24. Z. Bay, "Calculation of decay times from coincidence experiments," Phys. Rev. 77(3), 419-419 (1950).

25. G. Vicidomini et al., "Sharper low-power STED nanoscopy by time gating," Nat. Methods 8(7), 571-573 (2011).

26. J. Widengren et al., "Single-molecule detection and identification of multiple species by multiparameter fluorescence detection," Anal. Chem. 78(6), 2039-2050 (2006).

27. L. H. Chao et al., "A mechanism for tunable autoinhibition in the structure of a human $\mathrm{Ca}^{2+}$ /calmodulin-dependent kinase II holoenzyme," Cell 146(5), 732-745 (2011).

28. A. Hudmon et al., "A mechanism for $\mathrm{Ca}^{2+} /$ calmodulin-dependent protein kinase ii clustering at synaptic and nonsynaptic sites based on self-association," J. Neurosci. 25(30), 6971-6983 (2005).

29. M. H. Ulbrich and E. Y. Isacoff, "Rules of engagement for NMDA receptor subunits," Proc. Natl. Acad. Sci. 105(37), 14163-14168 (2008).

30. P. Opazo et al., "CaMKII triggers the diffusional trapping of surface AMPARs through phosphorylation of stargazin," Neuron 67(2), 239252 (2010). 
31. J. S. Ferreira et al., "Co-agonists differentially tune GluN2BNMDA receptor trafficking at hippocampal synapses," Elife 6, e25492 (2017).

32. M. Lemieux et al., "Translocation of CaMKII to dendritic microtubules supports the plasticity of local synapses," J. Cell Biol. 198(6), 10551073 (2012).

33. A. S. Leonard et al., "Calcium/calmodulin-dependent protein kinase II is associated with the N- methyl-D-aspartate receptor," Proc. Natl. Acad. Sci. U. S. A. 96(6), 3239-3244 (1999).

34. A. Barria and R. Malinow, "NMDA receptor subunit composition controls synaptic plasticity by regulating binding to CaMKII," Neuron 48(2), 289-301 (2005).

35. F. Gardoni et al., " $\alpha \mathrm{CaMKII}$ binding to the C-terminal tail of NMDA receptor subunit NR2A and its modulation by autophosphorylation," FEBS Lett. 456(3), 394-398 (1999).

36. K. U. Bayer et al., "Interaction with the NMDA receptor locks CaMKII in an active conformation," Nature 411(6839), 801-805 (2001).

37. K. U. Bayer et al., "Transition from reversible to persistent binding of CaMKII to postsynaptic sites and NR2B," J. Neurosci. 26(4), 11641174 (2006).

38. S. Tomita et al., "Stargazin modulates AMPA receptor gating and trafficking by distinct domains," Nature 435(7045), 1052-1058 (2005).

39. S. Tomita et al., "Dynamic interaction of stargazin-like TARPs with cycling AMPA receptors at synapses," Science 303(5663), 15081511 (2004).

40. A. Sumioka, D. Yan, and S. Tomita, "TARP phosphorylation regulates synaptic AMPA receptors through lipid bilayers," Neuron 66(5), 755767 (2010).

41. C. Bats, L. Groc, and D. Choquet, "The interaction between stargazin and PSD-95 regulates AMPA receptor surface trafficking," Neuron 53(5), 719-734 (2007).

42. L. Chen et al., "Stargazin regulates synaptic targeting of AMPA receptors by two distinct mechanisms," Nature 408(6815), 936-943 (2000).

43. A. Constals et al., "Glutamate-induced AMPA receptor desensitization increases their mobility and modulates short-term plasticity through unbinding from stargazin," Neuron 85(4), 787-803 (2015).

44. D. Nair et al., "Super-resolution imaging reveals that AMPA receptors inside synapses are dynamically organized in nanodomains regulated by PSD95," J. Neurosci. 33(32), 13204-13224 (2013).

45. H. E. Lu et al., "Multiple spatial and kinetic subpopulations of CaMKII in spines and dendrites as resolved by single-molecule tracking PALM," J. Neurosci. 34(22), 7600-7610 (2014).

46. H. D. MacGillavry et al., "Nanoscale scaffolding domains within the postsynaptic density concentrate synaptic ampa receptors," Neuron 78(4), 615-622 (2013).

47. T. Niehörster et al., "Multi-target spectrally resolved fluorescence lifetime imaging microscopy," Nat. Methods 13(3), 257-262 (2016).

48. J. R. Lakowicz et al., "Fluorescence lifetime imaging," Anal. Biochem. 202(2), 316-330 (1992).

49. Y. Sun et al., "Förster resonance energy transfer microscopy and spectroscopy for localizing protein-protein interactions in living cells," Cytom. Part A 83(9), 780-793 (2013).

50. P. König et al., "FRET-CLSM and double-labeling indirect immunofluorescence to detect close association of proteins in tissue sections," Lab. Invest. 86(8), 853-864 (2006).

51. Y. Wu et al., "Quantitative determination of spatial protein-protein correlations in fluorescence confocal microscopy," Biophys. J. 98(3), 493-504 (2010).

52. S. Padilla-Parra et al., "Quantitative comparison of different fluorescent protein couples for fast FRET-FLIM acquisition," Biophys. J. 97(8), 2368-2376 (2009).

53. M. Peter et al., "Multiphoton-FLIM quantification of the EGFP-mRFP1 FRET pair for localization of membrane receptor-kinase interactions," Biophys. J. 88(2), 1224-1237 (2005).

54. H. Murakoshi, S. J. Lee, and R. Yasuda, "Highly sensitive and quantitative FRET-FLIM imaging in single dendritic spines using improved non-radiative YFP," Brain Cell Biol. 36(1-4), 31-42 (2008).

55. J. Oracz et al., "Photobleaching in STED nanoscopy and its dependence on the photon flux applied for reversible silencing of the fluorophore," Sci. Rep. 7(1), 11354 (2017).
56. C. Li et al., "Recent research on stimulated emission depletion microscopy for reducing photobleaching," J. Microsc. 271, 4-16 (2018).

57. K. Kim et al., "Interplay of enzymatic and structural functions of CaMKII in long-term potentiation," J. Neurochem. 139(6), 959-972 (2016).

58. K. Shen and T. Meyer, "Dynamic control of CaMKII translocation and localization in hippocampal neurons by NMDA receptor stimulation," Science 284(5411), 162-167 (1999).

59. J. D. Petersen et al., "Distribution of postsynaptic density (PSD)-95 and $\mathrm{Ca}^{2+} /$ calmodulin-dependent protein kinase II at the PSD," J. Neurosci. 23(35), 11270-11278 (2003).

60. A. H. Tang et al., "A trans-synaptic nanocolumn aligns neurotransmitter release to receptors," Nature 536(7615), 210-214 (2016).

61. S. Galiani et al., "Strategies to maximize the performance of a STED microscope," Opt. Express 20(7), 7362-7374 (2012).

62. B. L. Sabatini, K. S. Thomas, and A. Pologruto, "ScanImage: flexible software for operating laser scanning microscopes," Biomed. Eng. Online 2, 13 (2003).

63. A. E. Siegman, Lasers, University Science Books, Sausalito, California (1986).

64. S. Labrecque et al., "Hyperspectral multiplex single-particle tracking of different receptor subtypes labeled with quantum dots in live neurons," J. Biomed. Opt. 21(4), 46008 (2016).

65. F. Nault and P. De Koninck in Protocols for Neural Cell Culture, L. Doering, Ed., pp. 137-159, Humana Press, New York (2009).

66. F. Lavoie-Cardinal et al., "Gold nanoparticle-assisted all optical localized stimulation and monitoring of $\mathrm{Ca}^{2+}$ signaling in neurons," Sci. Rep. 6(Oct 2015), 20619 (2016).

67. T. Staudt et al., "2, 2'-thiodiethanol: a new water soluble mounting medium for high resolution optical microscopy," Microsc. Res. Tech. 70(1), 1-9 (2007).

Christian Tardif is a PhD candidate in biophotonics. He received his BSc degree in physics engineering and his MSc degree in neurosciences from the Université Laval, Canada. He joined INO in Québec City in 2015. His work focuses on the development of new microscopy tools. His main interests are super-resolution microscopy, fluorescence lifetime imaging, neuroscience, and image analysis.

Gabriel Nadeau received his BSc degree in biomedical sciences and his MSc degree in biophotonics from the Université Laval, Canada. Currently, he is studying for a medicine degree at the Université Laval. His research interests are focused on neuronal calcium signaling, fluorescence imaging of biological tissues, and image processing.

Simon Labrecque received his MSc degree in physics and his $\mathrm{PhD}$ in biophotonics from the Universite Laval, Canada. He joined the CERVO Brain Research Center as an optical engineer and a coordinator in the Neurophotonics Center for the testing platform of new imaging tools and image analysis routines. He has a strong interest in cellular neuroscience, new developments in live optical microscopy, and single-molecule tracking with fluorescent molecules or nanoparticles.

Daniel Côté received his BSc degree in physics from Université Laval, Canada, in 1995 and his PhD in physics from the University of Toronto, Canada, in 2003. Following postdoctoral fellowships at the Ontario Cancer Institute and the Wellman Center for Photomedicine in Boston, he joined Université Laval where he is a full professor and affiliated with the CERVO Brain Research Center. His research group develops microscopy tools for neuroscience and neurosurgical guidance devices and investigates strategies for deciphering brain connectivity and function.

Flavie Lavoie-Cardinal received her $\mathrm{PhD}$ in chemistry from the University of Siegen, Germany in 2011, and postdoctoral trainings (i) at the MPI for biophysical chemistry in Göttingen, Germany until 2014, and (ii) at the CERVO Brain Research Center, Quebec City, Canada. Since 2017, she is an independent researcher at CERVO and an adjunct professor at Université Laval. Her research interests 
are in optical nanoscopy, cellular and molecular neuroscience, and application of machine learning to bioimaging techniques.

Paul De Koninck received his $\mathrm{PhD}$ in neurobiology from McGill University, Canada, in 1995 and postdoctoral training at Stanford University. In 2001, he joined the Department of Biochemistry,
Microbiology, and Bioinformatics at the Université Laval, Canada, where he is a full professor. $\mathrm{He}$ is the director of the cellular and molecular neuroscience division at the CERVO Brain Research Center in Quebec City. His research interests are in cellular and molecular neuroscience, synaptic signaling and plasticity, using advanced optical methods. 